\title{
角修正型双圆环面二次包络环面蜗杆传动的 啮合原理
}

\author{
赵亚平 ${ }^{\mathbb{*} *}, \mathrm{SU}$ DaiZhong ${ }^{\circledR}$, 张昭 ${ }^{\circledR}$, 魏文军, 董学朱 ${ }^{\circledR}$ \\ (1) 武汉科技大学机械自动化学院, 武汉 430081; \\ (2) Advanced Design and Manufacturing Engineering Center, Nottingham Trent University, Nottingham NG1 4BU, UK; \\ (3) 中国农业大学工学院, 北京 100083 \\ *E-mail: zhaoyaping1975@126.com
}

收稿日期: 2009-05-04; 接受日期: 2009-11-11

国家自然科学基金(批准号: 50705068, 50810105045), 湖北省自然科学基金(批准号：2007ABA282)和武汉科技大学科学研究重点项目(批 准号: 2006XZ6)资助

摘要导出了一般化的角修形条件, 解释了角修形的物理意义; 提出啮合分析的法截面法, 该 方法可以用来计算奇异啮合点附近共轭齿面偶的法向距离; 运用该方法, 通过分析法截线, 指明 角修形传动蜗杆螺旋面、蜗轮齿面名义原接触区、新接触区三者沿传动副瞬时接触线奇点轨迹相 互交叉, 说明三者虽然相互密切, 但程度不同; 阐明角修形切去蜗轮齿面二次接触区, 并且使得 蜗杆工作长度变短的机理. 在角修正型二包传动啮合理论的指导下, 对角修形双圆环面二包传动 的啮合特性进行了深入系统的研究, 发现这是一种性能优良的新型环面蜗杆传动装置.

关键词 环面蜗杆 角修形 二次包络

密切 法截线
双圆环面二次包络环面蜗杆传动, 以圆环面作 为蜗杆螺旋面的产形母面, 可以同时加工蜗杆齿槽 的两侧螺旋面; 蜗轮齿面由产形面与蜗杆螺旋面一 致的环面滚刀范成. 除了出色的制造工艺性和能够 应用于多头数小传动比的场合外, 双圆环面二包传 动还具备一系列的优良啮合特性, 例如蜗杆双线工 作区长, 蜗轮齿面接触区宽阔且瞬时接触线分布合 理, 诱导主曲率值合乎等强度原则及润滑特性优良 等 ${ }^{[1 \sim 3]}$.

不过, 与传统二次包络环面蜗杆传动相似, 双圆 环面二包副蜗轮齿面中部也存在二次接触区, 该区 域内瞬时接触线相互交叉, 蜗杆蜗轮接触频率高, 容 易发生疲劳点蚀, 因此, 二次接触区是蜗轮齿面的薄 弱部位, 制约着传动副承载能力的充分发挥与进一
步提高. 解决这个问题的办法之一, 就是对蜗杆进行 角修形, 使得二次接触区在蜗轮齿面上不再出现. 由 此，可以得到角修正双圆环面二包传动.

20 世纪 70 年代, 日本学者率先开展了角修形环 面蜗杆副的研究 ${ }^{[4]}$; 随后, 我国学者按日本学者的思 路, 从数学上对这个问题进行了详细、严格地证明与 诠释 ${ }^{[5]}$; 文献[6 8]研究了直平面角修形二包传动, 实 际上这是角修形传动的一个特款; 文献[9]研究了双 雉面角修形二包传动; 最近, 文献[10]对环面蜗杆传 动角修形进行了较为全面的研究.

本文所述的角修正双圆环面二包传动的制造与 相应的标准传动大致相同, 主要不同之处存在于蜗 杆制造阶段. 具体陈述如下.

刀座轴线正向与蜗杆毛坏轴线正向夹角 $90^{\circ}+\Delta \Sigma$ 
不是 $90^{\circ}$, 刀座轴线与蜗杆毛坏轴线间工艺中心距为 $a_{\mathrm{d}}$ 不是蜗杆副中心距 $a$, 蜗杆毛坏与刀座间工艺传动 比为 $i_{1 \mathrm{~d}}$ 不是蜗杆副速比 $i_{12}$. 刀座沿自身轴线作螺旋 运动, 螺旋参数为 $p_{\mathrm{d}}$. 这也是角修形双圆环面二包传 动与角修形直平面二包传动的不同之处. 作为修形 参数的 $\Delta \Sigma, a_{\mathrm{d}}, i_{1 \mathrm{~d}}$ 和 $p_{\mathrm{d}}$ 需按一定关系确定.

啮合分析表明，角修正双圆环面二次包络环面 蜗杆副具备许多优良特性, 是一种很有前途的交叉 轴间传动装置.

\section{1 产形砂轮设计与蜗杆螺旋面的方程}

\section{1 产形砂轮的设计原理}

在砂轮轴截面中建立坐标系 $\left(O_{\mathrm{a}} ; x, y\right)$, 如图 1 所 示. $x$ 轴位于砂轮中间平面内, $y$ 轴沿砂轮轴线, 此轴 截面交砂轮产形圆环面于 $\overparen{A B}$. 若砂轮半径为 $r_{\mathrm{d}}$ 且其 顶宽为 $s_{\mathrm{a}}$, 则 $A$ 点坐标为 $x_{A}=r_{\mathrm{d}}, y_{A}=s_{\mathrm{a}} / 2$. 考虑到砂 轮在刀座上安装后与蜗杆毛坏的相对位置, $B$ 点的横 坐标为 $x_{B}=r_{\mathrm{d}}-h_{\mathrm{f}}-r_{2}\left(1-\cos \psi_{0}\right)$, 其中 $r_{2}$ 为蜗轮分圆 半径, $h_{\mathrm{f}}$ 为蜗杆副齿根高, $\psi_{0}$ 为蜗杆分圆齿槽宽半角.

图 1 中, 点 $O(p, q)$ 为 $\overparen{A B}$ 的圆心, 点 $C$ 为弦 $A B$ 的 中点, 根据几何关系, 可以得到直线 $B O$ 和 $C O$ 的方 程分别为

$$
\left\{\begin{array}{l}
x-y \tan \alpha_{\mathrm{d}}-x_{B}+y_{B} \tan \alpha_{\mathrm{d}}=0, \\
8\left(r_{\mathrm{d}}-x_{B}\right) x-4\left(2 y_{B}-s_{\mathrm{a}}\right) y-s_{\mathrm{a}}^{2}+4 x_{B}^{2}+4 y_{B}^{2}-4 r_{\mathrm{d}}^{2}=0,
\end{array}\right.
$$

式中, $\alpha_{\mathrm{d}}$ 为砂轮工作齿形角, $\alpha_{\mathrm{d}}=\arctan \left(\tan \alpha_{\mathrm{t}} \cos \beta\right)$, $\alpha_{\mathrm{t}}$ 为蜗杆轴向压力角, $\beta$ 为砂轮轴线倾角; $y_{B}$ 为 $B$ 点纵 坐标.

解方程组(1), 可以得到点 $O$ 的坐标为

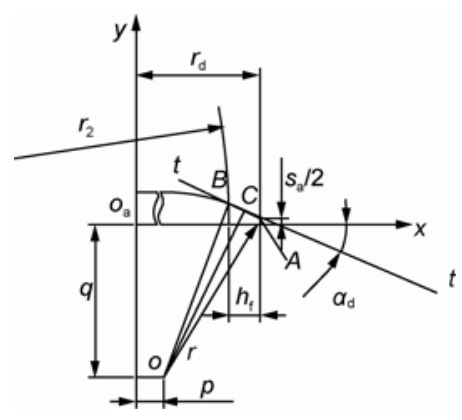

图 1 砂轮轴截面内圆弧工作廓线的几何特性

$$
\left\{\begin{array}{l}
p=\frac{s_{\mathrm{a}}^{2} \tan \alpha_{\mathrm{d}}+P_{1} s_{\mathrm{a}}+P_{2}}{4\left(s_{\mathrm{a}}-2 x_{B} \tan \alpha_{\mathrm{d}}-2 y_{B}+2 r_{\mathrm{d}} \tan \alpha_{\mathrm{d}}\right)}, \\
q=\frac{s_{\mathrm{a}}^{2}+Q}{4\left(s_{\mathrm{a}}-2 x_{B} \tan \alpha_{\mathrm{d}}-2 y_{B}+2 r_{\mathrm{d}} \tan \alpha_{\mathrm{d}}\right)},
\end{array}\right.
$$

式中,

$\left\{\begin{array}{l}P_{1}=4\left(x_{B}-y_{B} \tan \alpha_{\mathrm{d}}\right), \\ P_{2}=-4 x_{B}^{2} \tan \alpha_{\mathrm{d}}+4 y_{B}^{2} \tan \alpha_{\mathrm{d}}-8 x_{B} y_{B}+4 r_{\mathrm{d}}^{2} \tan \alpha_{\mathrm{d}}, \\ Q=4 x_{B}^{2}-4 y_{B}^{2}-8 x_{B} y_{B} \tan \alpha_{\mathrm{d}}-8 r_{\mathrm{d}} x_{B}+8 r_{\mathrm{d}} y_{B} \tan \alpha_{\mathrm{d}}+4 r_{\mathrm{d}}^{2} .\end{array}\right.$

考虑到 $A$ 点在 $\overparen{A B}$ 上, 所以

$$
\left(p-r_{\mathrm{d}}\right)^{2}+\left(q-\frac{s_{\mathrm{a}}}{2}\right)^{2}-\rho^{2}=0,
$$

式中, $\rho$ 为砂轮轴截面内工作廓线 $\overparen{A B}$ 的半径.

当给定砂轮顶宽后，把(2)式代入(3)式可以得到 关于 $y_{B}$ 的四次代数方程, 解得的 $y_{B}$ 值应满足 $0<y_{B} \leqslant r_{2} \sin \psi_{0}$, 以保证砂轮能顺利插入蜗杆齿槽. 待确定 $y_{B}$ 值后，由(2)式可以确定 $p$ 和 $q$ 的值，从而完 成砂轮产形面的设计.

\section{2 产形圆环面方程与其特性参数}

坐标系 $\sigma_{\mathrm{a}}\left(o_{\mathrm{a}} ; \boldsymbol{i}_{\mathrm{a}}, \boldsymbol{j}_{\mathrm{a}}, \boldsymbol{k}_{\mathrm{a}}\right)$ 与砂轮相固连, 砂轮的任 意轴截面如图 2 所示, 单位向量 $\boldsymbol{k}_{\mathrm{a}}$ 沿砂轮轴线方向, 单位向量 $\boldsymbol{i}_{\mathrm{a}}$ 和 $\boldsymbol{j}_{\mathrm{a}}$ 在砂轮中间平面内. 利用圆向量函数 与球向量函数 ${ }^{[11]}$, 可以写出产形圆环面 $\Sigma_{\mathrm{d}}$ 的矢量方 程为

$$
\left(\boldsymbol{r}_{\mathrm{a}}\right)_{\mathrm{a}}=p \boldsymbol{e}_{\mathrm{a}}(\theta)+q \boldsymbol{k}_{\mathrm{a}}+\rho \boldsymbol{m}_{\mathrm{a}}(\theta, \phi)=x_{\mathrm{a}} \boldsymbol{i}_{\mathrm{a}}+y_{\mathrm{a}} \boldsymbol{j}_{\mathrm{a}}+z_{\mathrm{a}} \boldsymbol{k}_{\mathrm{a}},
$$
式中 $x_{\mathrm{a}}=(\rho \sin \phi+p) \cos \theta, y_{\mathrm{a}}=(\rho \sin \phi+p) \sin \theta, z_{a}=$ $\rho \cos \phi+q, \phi$ 和 $\theta$ 是 $\Sigma_{\mathrm{d}}$ 的两个参数.

产形圆环面 $\Sigma_{\mathrm{d}}$ 为回转曲面, 利用微分几何中的 经典方法, 容易得到其单位法向量、两个主曲率和相 应的两个主方向分别为

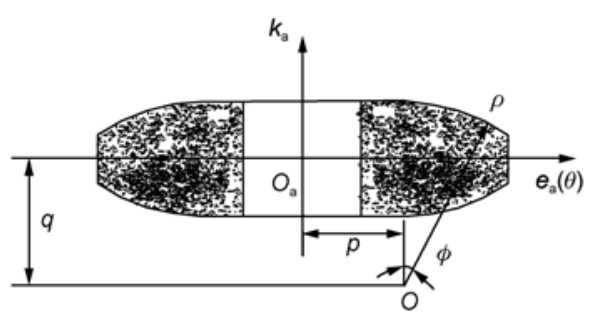

图 $2 \sigma_{\mathrm{a}}$ 中的砂轮轴截面 


$$
\left\{\begin{array}{l}
(\boldsymbol{n})_{\mathrm{a}}=\boldsymbol{m}_{\mathrm{a}}(\theta, \phi), \\
k_{1}=-\frac{1}{\rho} \\
k_{2}=-\frac{\sin \phi}{\rho \sin \phi+p} \\
\left(\boldsymbol{g}_{1}\right)_{\mathrm{a}}=\boldsymbol{n}_{\mathrm{a}}(\theta, \phi), \\
\left(\boldsymbol{g}_{2}\right)_{\mathrm{a}}=\boldsymbol{g}_{\mathrm{a}}(\theta) .
\end{array}\right\}
$$

产形圆环面 $\Sigma_{\mathrm{d}}$ 的单位法向量 $(\boldsymbol{n})$ a 是由砂轮的实 体指向空域.

动坐标系 $\sigma_{\mathrm{d}}\left(o_{\mathrm{d}} ; \boldsymbol{i}_{\mathrm{d}}, \boldsymbol{j}_{\mathrm{d}}, \boldsymbol{k}_{\mathrm{d}}\right)$ 与刀座相固连, $\boldsymbol{k}_{\mathrm{d}}$ 轴沿 刀座轴线方向, $\sigma_{\mathrm{a}}$ 与 $\sigma_{\mathrm{d}}$ 的相对位置如图 3 所示. 通过 坐标变换, 在 $\sigma_{\mathrm{d}}$ 中可以得到 $\Sigma_{\mathrm{a}}$ 的方程及其单位法向 量和两个主方向分别为

$$
\begin{aligned}
\left\{\begin{array}{l}
\left(\boldsymbol{r}_{\mathrm{d}}\right)_{\mathrm{d}}=R\left[\boldsymbol{i}_{\mathrm{d}},-\left(90^{\circ}-\beta\right)\right]\left(\boldsymbol{r}_{\mathrm{a}}\right)_{\mathrm{a}}-a_{0} \boldsymbol{i}_{\mathrm{d}}=x_{\mathrm{d}} \boldsymbol{i}_{\mathrm{d}}+y_{\mathrm{d}} \boldsymbol{j}_{\mathrm{d}}+z_{\mathrm{d}} \boldsymbol{k}_{\mathrm{d}}, \\
(\boldsymbol{n})_{\mathrm{d}}=R\left[\boldsymbol{i}_{\mathrm{d}},-\left(90^{\circ}-\beta\right)\right](\boldsymbol{n})_{\mathrm{a}}=n_{x} \boldsymbol{i}_{\mathrm{d}}+n_{y} \boldsymbol{j}_{\mathrm{d}}+n_{z} \boldsymbol{k}_{\mathrm{d}}, \\
x_{\mathrm{d}}=x_{\mathrm{a}}-a_{0}, \\
y_{\mathrm{d}}=y_{\mathrm{a}} \sin \beta+z_{\mathrm{a}} \cos \beta, \\
z_{\mathrm{d}}=-y_{\mathrm{a}} \cos \beta+z_{\mathrm{a}} \sin \beta, \\
n_{x}=\sin \phi \cos \theta, \\
n_{y}=\sin \beta \sin \phi \sin \theta+\cos \beta \cos \phi, \\
n_{\mathrm{z}}=-\cos \beta \sin \phi \sin \theta+\sin \beta \cos \phi,
\end{array}\right\} \\
\left.\qquad \begin{array}{l}
\left(\boldsymbol{g}_{m}\right)_{2}=R\left[\boldsymbol{i}_{2},-\left(90^{\circ}-\beta\right)\right]\left(\boldsymbol{g}_{m}\right)_{\mathrm{a}} \\
=g_{m x} \boldsymbol{i}_{2}+g_{m y} \boldsymbol{j}_{2}+g_{m z} \boldsymbol{k}_{2}, \quad(m=1,2), \\
g_{1 x}=\cos \phi \cos \theta, \\
g_{1 y}=\sin \beta \cos \phi \sin \theta-\cos \beta \sin \phi, \\
g_{1 z}=-\cos \beta \cos \phi \sin \theta-\sin \beta \sin \phi, \\
g_{2 x}=-\sin \theta, \\
g_{2 y}=\sin \beta \cos \theta, \\
g_{2 z}=-\cos \beta \cos \theta,
\end{array}\right\}
\end{aligned}
$$

式中, $a_{0}=a_{\mathrm{d}}-r_{\mathrm{f} 1}-r_{\mathrm{d}}, r_{\mathrm{f} 1}$ 为蜗杆喉部根圆半径, $a_{\mathrm{d}}=$ $a+\Delta a, \Delta a$ 为角修形中心距变动量; $R\left[\boldsymbol{i}_{\mathrm{d}},-\left(90^{\circ}-\beta\right)\right]$

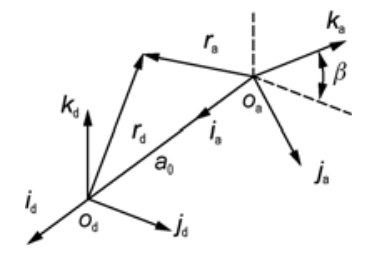

图 3 坐标系 $\sigma_{\mathrm{a}}$ 和 $\sigma_{\mathrm{d}}$ 的相对位置
为绕 $\boldsymbol{i}_{\mathrm{d}}$ 轴的旋转变换矩阵 ${ }^{[11]}$.

\section{3 产形面族与一次包络的相对运动}

如图 4 所示, 静坐标系 $\sigma_{o \mathrm{~d}}\left(o_{o \mathrm{~d}} ; \boldsymbol{i}_{o \mathrm{~d}}, \boldsymbol{j}_{o \mathrm{~d}}, \boldsymbol{k}_{o \mathrm{~d}}\right)$ 和 $\sigma_{o 1}\left(o_{1} ; \boldsymbol{i}_{o 1}, \boldsymbol{j}_{o 1}, \boldsymbol{k}_{o 1}\right)$ 分别表示刀座和蜗杆毛坏的初始 位置. 向量 $\boldsymbol{k}_{o \mathrm{~d}}$ 和 $\boldsymbol{k}_{o 1}$ 分别沿刀座和蜗杆毛坏的轴线 方向, 向量 $\boldsymbol{i}_{o \mathrm{~d}}$ 和 $\boldsymbol{i}_{o 1}$ 沿着它们的公垂线方向, $\left|\overline{O_{1} O_{o \mathrm{~d}}}\right|=$ $a_{\mathrm{d}}$.

建立动坐标系 $\sigma_{1}\left(o_{1} ; \boldsymbol{i}_{1}, \boldsymbol{j}_{1}, \boldsymbol{k}_{1}\right)$ 与环面蜗杆毛坏相 固连. 刀座作螺旋运动, 当它绕自身轴线 $\boldsymbol{k}_{o \mathrm{~d}}$ 转过角 度 $\varphi_{\mathrm{d}}$, 蜗杆毛坏绕其轴线 $\boldsymbol{k}_{o 1}$ 转过角度 $\varphi=i_{1 \mathrm{~d}} \varphi_{\mathrm{d}}$; 与 此同时, 刀座沿其轴线 $\boldsymbol{k}_{o \mathrm{~d}}$ 移动的距离是 $\left|\overline{O_{o \mathrm{~d}} O_{\mathrm{d}}}\right|=p_{\mathrm{d}} \varphi$.

刀座作螺旋运动的过程中, 在静系 $\sigma_{o \mathrm{~d}}$ 中形成单 参数曲面族 $\left\{\Sigma_{\mathrm{d}}\right\}$. 由(6)式, 经过坐标变换, 可以得到 $\left\{\Sigma_{\mathrm{d}}\right\}$ 的矢量方程及相应单位法向量族的方程分别为

$$
\left\{\begin{array}{l}
\left(\boldsymbol{r}_{\mathrm{d}}\right)_{o \mathrm{~d}}=R\left[\boldsymbol{k}_{o \mathrm{~d}}, \varphi_{\mathrm{d}}\right]\left(\boldsymbol{r}_{\mathrm{d}}\right)_{\mathrm{d}}=x_{o \mathrm{~d}} \boldsymbol{i}_{o \mathrm{~d}}+y_{o \mathrm{~d}} \boldsymbol{j}_{o \mathrm{~d}}+z_{\mathrm{d}} \boldsymbol{k}_{o \mathrm{~d}}, \\
(\boldsymbol{n})_{o \mathrm{~d}}=R\left[\boldsymbol{k}_{o \mathrm{~d}}, \varphi_{\mathrm{d}}\right](\boldsymbol{n})_{\mathrm{d}}=n_{o x} \boldsymbol{i}_{o \mathrm{~d}}+n_{o y} \boldsymbol{j}_{o \mathrm{~d}}+n_{z} \boldsymbol{k}_{o \mathrm{~d}},
\end{array}\right\}
$$

式中, $R\left[\boldsymbol{k}_{o \mathrm{~d}}, \varphi_{\mathrm{d}}\right]$ 为绕 $\boldsymbol{k}_{o \mathrm{~d}}$ 轴的旋转变换矩阵.

假设蜗杆毛坏绕其轴线转动的角速度为 $|\omega|=$ $1 \mathrm{rad} / \mathrm{s}$, 则刀座绕其轴线转动的角速度为 $\left|\omega_{\mathrm{d}}\right|=1 / i_{1 \mathrm{~d}}$ $\mathrm{rad} / \mathrm{s}$. 于是, 在静坐标系 $\sigma_{o \mathrm{~d}}$ 中, 得到刀座与蜗杆毛 坏相对运动角速度矢量和相对运动速度矢量分别为

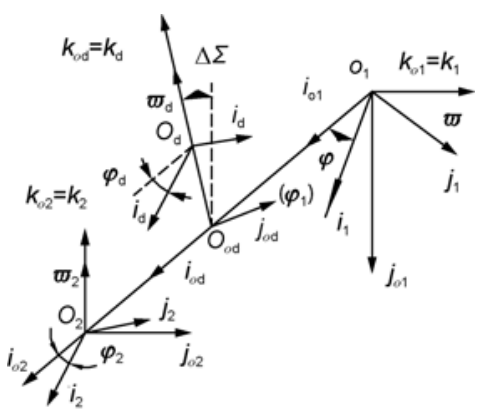

图 4 坐标系 


$$
\left\{\begin{array}{l}
\left(\boldsymbol{\omega}_{\mathrm{d} 1}\right)_{o \mathrm{~d}}=-\cos \Delta \Sigma \boldsymbol{j}_{o \mathrm{~d}}+\left(\frac{1}{i_{\mathrm{ld}}}+\sin \Delta \Sigma\right) \boldsymbol{k}_{o \mathrm{~d}}, \\
\left(\boldsymbol{V}_{\mathrm{d} 1}\right)_{o \mathrm{~d}}=V_{\mathrm{d} 1}^{(x)} \boldsymbol{i}_{o \mathrm{~d}}+V_{\mathrm{d} 1}^{(y)} \boldsymbol{j}_{o \mathrm{~d}}+V_{\mathrm{d} 1}^{(z)} \boldsymbol{k}_{o \mathrm{~d}},
\end{array}\right.
$$

式中,

$$
\left\{\begin{aligned}
V_{\mathrm{d} 1}^{(x)}= & -y_{o \mathrm{~d}}\left(\frac{1}{i_{\mathrm{ld}}}+\sin \Delta \Sigma\right)-\left(z_{\mathrm{d}}+p_{\mathrm{d}} \varphi\right) \cos \Delta \Sigma \\
& +\frac{\mathrm{d} \Delta a}{\mathrm{~d} \varphi}, \\
V_{\mathrm{d} 1}^{(y)}= & x_{o \mathrm{~d}}\left(\frac{1}{i_{\mathrm{ld}}}+\sin \Delta \Sigma\right)+a_{\mathrm{d}} \sin \Delta \Sigma, \\
V_{\mathrm{d} 1}^{(z)}= & x_{o \mathrm{~d}} \cos \Delta \Sigma+a_{\mathrm{d}} \cos \Delta \Sigma+p_{\mathrm{d}} .
\end{aligned}\right.
$$

\section{4 一次包络的啮合函数与蜗杆螺旋面方程}

根据齿轮啮合理论中的定义, 由(8)和(9)式, 得 到一次包络的啮合函数为

$$
\begin{aligned}
\Phi_{\mathrm{d}} & =(\boldsymbol{n})_{o \mathrm{~d}} \cdot\left(\boldsymbol{V}_{\mathrm{d} 1}\right)_{o \mathrm{~d}} \\
& =A_{\mathrm{d}} \sin \left(\varphi / i_{\mathrm{ld}}\right)+B_{\mathrm{d}} \cos \left(\varphi / i_{\mathrm{ld}}\right)+C_{\mathrm{d}} \\
& =\Phi_{\mathrm{d}}(\phi, \theta, \varphi),
\end{aligned}
$$

式中,

$$
\left\{\begin{array}{l}
A_{\mathrm{d}}=n_{x} a_{\mathrm{d}} \sin \Delta \Sigma-n_{y}\left(\frac{\mathrm{d} \Delta a}{\mathrm{~d} \varphi}-p_{\mathrm{d}} \varphi \cos \Delta \Sigma\right)-T_{x} \cos \Delta \Sigma, \\
B_{\mathrm{d}}=n_{x}\left(\frac{\mathrm{d} \Delta a}{\mathrm{~d} \varphi}-p_{\mathrm{d}} \varphi \cos \Delta \Sigma\right)+n_{y} a_{\mathrm{d}} \sin \Delta \Sigma-T_{y} \cos \Delta \Sigma, \\
C_{\mathrm{d}}=n_{z}\left(p_{\mathrm{d}}+a_{\mathrm{d}} \cos \Delta \Sigma\right)+T_{z}\left(\frac{1}{i_{\mathrm{dd}}}+\sin \Delta \Sigma\right), \\
T_{x}=\left|\begin{array}{ll}
y_{\mathrm{d}} & z_{\mathrm{d}} \\
n_{y} & n_{z}
\end{array}\right|, \\
T_{y}=-\left|\begin{array}{ll}
x_{\mathrm{d}} & z_{\mathrm{d}} \\
n_{x} & n_{z}
\end{array}\right|, \\
T_{z}=\left|\begin{array}{ll}
x_{\mathrm{d}} & y_{\mathrm{d}} \\
n_{x} & n_{y}
\end{array}\right| .
\end{array}\right\},
$$

由于一次包络啮合函数的系数 $A_{\mathrm{d}}$ 与 $B_{\mathrm{d}}$ 是运动参 数 $\varphi$ 的函数, 所以双圆环面二包角修形属于变参数修 形, 或称动修形.

由(8)和(10)式, 在坐标系 $\sigma_{1}$ 中, 可以得到蜗杆螺 旋面 $\Sigma_{1}$ 的方程及其单位法向量分别为

$$
\begin{aligned}
& \left.\begin{array}{rl}
\left(\boldsymbol{r}_{1}\right)_{1} & \left.=R\left[\boldsymbol{k}_{1},-\varphi\right]\left\{R\left[\boldsymbol{i}_{o 1}, 90^{\circ}+\Delta \Sigma\right]\left[\begin{array}{c}
x_{o \mathrm{~d}} \\
y_{o \mathrm{~d}} \\
z_{\mathrm{d}}+p_{\mathrm{d}} \varphi
\end{array}\right]+a_{\mathrm{d}} \boldsymbol{i}_{o 1}\right\}\right\} \\
& =x_{o 1} \boldsymbol{e}_{1}(-\varphi)+y_{o 1} \boldsymbol{g}_{1}(-\varphi)+z_{1} \boldsymbol{k}_{1}, \\
\Phi_{\mathrm{d}}= & 0,
\end{array}\right\} \\
& \left\{\begin{array}{l}
x_{o 1}=x_{o \mathrm{~d}}+a_{\mathrm{d}}, \\
y_{o 1}=-y_{o \mathrm{~d}} \sin \Delta \Sigma-\left(z_{\mathrm{d}}+p_{\mathrm{d}} \varphi\right) \cos \Delta \Sigma, \\
z_{1}=y_{o \mathrm{~d}} \cos \Delta \Sigma-\left(z_{\mathrm{d}}+p_{\mathrm{d}} \varphi\right) \sin \Delta \Sigma,
\end{array}\right\} \\
& \left\{\begin{aligned}
(\boldsymbol{n})_{1} & =R\left[\boldsymbol{k}_{1},-\varphi\right] R\left[\boldsymbol{i}_{o 1}, 90^{\circ}+\Delta \Sigma\right](\boldsymbol{n})_{o \mathrm{~d}} \\
& =n_{o x} \boldsymbol{e}_{1}(-\varphi)+n_{o 1}^{(y)} \boldsymbol{g}_{1}(-\varphi)+n_{1 z} \boldsymbol{k}_{1}, \\
n_{o 1}^{(y)} & =-n_{o y} \sin \Delta \Sigma-n_{z} \cos \Delta \Sigma, \\
n_{1 z}= & n_{o y} \cos \Delta \Sigma-n_{z} \sin \Delta \Sigma,
\end{aligned}\right\}
\end{aligned}
$$

式中, $R\left[\boldsymbol{i}_{o 1}, 90^{\circ}+\Delta \Sigma\right]$ 和 $R\left[\boldsymbol{k}_{1},-\varphi\right]$ 分别为绕 $\boldsymbol{i}_{o 1}$ 轴和绕 $\boldsymbol{k}_{1}$ 轴的旋转变换矩阵.

单位法向量 $(\boldsymbol{n})_{1}$ 的方向是由空域指向蜗杆实体.

(11)式中, 有 $\phi, \theta$ 和 $\varphi$ 三个参数, 但其中只有两个 互相独立, 作为蜗杆螺旋面 $\Sigma_{1}$ 的参数. 给定不同的 $\varphi$ 值, 由(11)式, 可以得到蜗杆螺旋面 $\Sigma_{1}$ 的一族特征线.

由蜗杆螺旋面方程式(11), 可以算得蜗杆轴截面 内喉部对称齿分度圆弦齿厚 $\vec{s}_{1}$. 若相应的理论弦齿 厚为 $\bar{s}_{1}$, 则可按 $\left|\bar{s}_{1}-\overline{s_{1}}\right|<0.02$ 确定砂轮顶宽 $s_{\mathrm{a}}$ 的值, 进而决定产形砂轮的其他参数. 由此可见, 角修正双 圆环面包络环面蜗杆产形砂轮的独立参数有三个, 分别是 $r_{\mathrm{d}}, \rho$ 和 $\beta$.

\section{2 角修形参数的确定}

如图 4 所示, 静坐标系 $\sigma_{o 1}$ 和 $\sigma_{o 2}\left(O_{2} ; \boldsymbol{i}_{o 2}, \boldsymbol{j}_{o 2}, \boldsymbol{k}_{o 2}\right)$ 分别表示蜗杆蜗轮的初始位置. $\boldsymbol{k}_{o 1}$ 和 $\boldsymbol{k}_{o 2}$ 分别沿蜗杆 蜗轮的轴线方向, 而且二者互相垂直, $\boldsymbol{i}_{o 1}$ 和 $\boldsymbol{i}_{o 2}$ 沿它们 的公垂线方向, 公垂线段 $\left|\overline{O_{1} O_{2}}\right|=a$. 动坐标系 $\sigma_{1}$ 和 $\sigma_{2}\left(O_{2} ; \boldsymbol{i}_{2}, \boldsymbol{j}_{2}, \boldsymbol{k}_{2}\right)$ 分别与蜗杆和蜗轮相固连. 当蜗杆绕 其轴线 $\boldsymbol{k}_{o 1}$ 的转角为 $\varphi_{1}$, 则蜗轮绕其轴线 $\boldsymbol{k}_{o 2}$ 的相应转 角为 $\varphi_{2}=\varphi_{1} / i_{12}$.

\section{1 蜗杆螺旋面族与二次包络的相对运动}

蜗杆与蜗轮的啮合过程中, 绕其轴线 $\boldsymbol{k}_{o 1}$ 作旋转 
运动, 在静坐标系 $\sigma_{o 1}$ 中形成单参数曲面族 $\left\{\Sigma_{1}\right\}$. 由 (11)和(12)式, 经过坐标变换, 得到 $\left\{\Sigma_{1}\right\}$ 的方程及相 应单位法向量族的方程为

$$
\left\{\begin{array}{l}
\left(\boldsymbol{r}_{1}^{*}\right)_{o 1}=R\left[\boldsymbol{k}_{o 1}, \varphi_{1}\right]\left(\boldsymbol{r}_{1}\right)_{1}=x_{o 1}^{*} \boldsymbol{i}_{o 1}+y_{o 1}^{*} \boldsymbol{j}_{o 1}+z_{1} \boldsymbol{k}_{o 1}, \\
\Phi_{\mathrm{d}}=0, \\
\left(\boldsymbol{n}^{*}\right)_{o 1}=R\left[\boldsymbol{k}_{o 1}, \varphi_{1}\right](\boldsymbol{n})_{1}, \\
x_{o 1}^{*}=x_{o 1} \cos \left(\varphi_{1}-\varphi\right)-y_{o 1} \sin \left(\varphi_{1}-\varphi\right), \\
y_{o 1}^{*}=x_{o 1} \sin \left(\varphi_{1}-\varphi\right)+y_{o 1} \cos \left(\varphi_{1}-\varphi\right),
\end{array}\right\}
$$

式中, $R\left[\boldsymbol{k}_{o 1}, \varphi_{1}\right]$ 为绕 $\boldsymbol{k}_{o 1}$ 轴的旋转变换矩阵.

同样地, 可以假设蜗杆绕其轴线转动的角速度 为 $\left|\omega_{1}\right|=1 \mathrm{rad} / \mathrm{s}$, 不失一般, 则蜗轮绕其轴线转动的 角速度为 $\left|\omega_{2}\right|=1 / i_{12} \mathrm{rad} / \mathrm{s}$. 于是, 在静坐标系 $\sigma_{o 1}$ 中, 得到蜗杆与蜗轮相对运动角速度矢量、相对运动速度 矢量分别为

$$
\left\{\begin{array}{l}
\left(\boldsymbol{\omega}_{12}\right)_{o 1}=\frac{1}{i_{12}} \boldsymbol{j}_{o 1}+\boldsymbol{k}_{o 1}, \\
\left(\boldsymbol{V}_{12}\right)_{o 1}=-\left(y_{o 1}^{*}-\frac{z_{1}}{i_{12}}\right) \boldsymbol{i}_{o 1}+x_{o 1}^{*} \boldsymbol{j}_{o 1}-\frac{x_{o 1}^{*}-a}{i_{12}} \boldsymbol{k}_{o 1} .
\end{array}\right.
$$

\section{2 二次包络的啮合函数与角修形参数的确定}

根据齿轮啮合理论中的定义, 由(13)和(14)式, 可以得到二次包络的啮合函数为 $\Phi=\left(\boldsymbol{n}^{*}\right)_{o 1} \cdot\left(\boldsymbol{V}_{12}\right)_{o 1}$. 按向量回转的理论 ${ }^{[2]}$ 进行计算, 并利用 $\Phi_{\mathrm{d}}=0$ 化简后, 得到

$$
\begin{aligned}
\Phi= & \Phi\left(\phi, \theta, \varphi, \varphi_{1}\right) \\
= & 2 \sin \frac{\varphi_{1}-\varphi}{2}\left(A \sin \frac{\varphi_{1}-\varphi}{2}+B \cos \frac{\varphi_{1}-\varphi}{2}\right) \\
& +C \cos \left(\varphi_{1}-\varphi\right),
\end{aligned}
$$

式中,

$$
\begin{aligned}
& C=\frac{T_{z}}{i_{12}}\left[\frac{i_{12}-\tan \Delta \Sigma}{i_{1 \mathrm{~d}}}-\frac{1}{\cos \Delta \Sigma}\right]+n_{o x}\left(1-\frac{\tan \Delta \Sigma}{i_{12}}\right) \frac{\mathrm{d} \Delta a}{\mathrm{~d} \varphi} \\
& -n_{o y} \frac{a \sin \Delta \Sigma+\Delta a}{i_{12} \cos \Delta \Sigma}+\frac{n_{z}}{i_{12}}\left[p_{\mathrm{d}}\left(i_{12}-\tan \Delta \Sigma\right)-a \sin \Delta \Sigma\right] .
\end{aligned}
$$

角修形条件下，应使 $C \equiv 0$. 但 $T_{z}, n_{o x}, n_{o y}$ 和 $n_{z}$ 都 是 $\phi, \theta$ 和 $\varphi$ 函数, 在蜗杆蜗轮啮合过程中, 不可能恒 为 0 . 不过它们的系数完全由角修形参数构成, 所以 只需使它们的系数同时为零, 就可保证 $C \equiv 0$.

由 $T_{z}$ 的系数为 0 , 可以得到

$$
\begin{aligned}
\Delta \Sigma & =-\arctan \frac{1}{i_{12}} \pm \arccos \frac{i_{1 \mathrm{~d}}}{\sqrt{i_{12}^{2}+1}} \text { 或 } \\
i_{\mathrm{ld}} & =i_{12} \cos \Delta \Sigma-\sin \Delta \Sigma .
\end{aligned}
$$

从(16)式可知, 若以 $i_{1 \mathrm{~d}}$ 为角修形基本变量, 确定 $\Delta \Sigma$, 可以避免在选配刀座与蜗杆毛坯间传动链的交换 齿轮时, 造成的工艺传动比误差; 但不利于控制角修 形量 $\Delta \Sigma$ 的大小. 与直平面二包角修形不同，双圆环面 二包角修形以 $\Delta \Sigma$ 与 $\beta$ 共同决定砂轮轴线与蜗杆毛坏 轴线的夹角. 这一点对于磨削多头数角修正环面蜗杆 极为有利. 虽然 $\Delta \Sigma$ 的值可正可负, 但是算例研究表 明，当 $\Delta \Sigma<0$ 时，容易造成环面蜗杆边齿顶减薄、变尖， 而且也不会对蜗杆副的啮合性能有显著的改善.

由 $n_{o x}$ 的系数为 0 , 可以得到 $\left(1-\tan \Delta \Sigma / i_{12}\right) \mathrm{d} \Delta a /$ $\mathrm{d} \varphi=0$. 该式有意义的解应是 $\mathrm{d} \Delta a / \mathrm{d} \varphi=0$, 即 $\Delta a=$ const. 由此可见, 加工角修正环面蜗杆的工艺 中心距为常数.

由 $n_{o y}$ 的系数为 0 , 可以得到 $\Delta a=-a \sin ^{2} \Delta \Sigma$, 说 明, $a_{\mathrm{d}}<a$.

由 $n_{z}$ 的系数为 0 , 可以得到

$$
p_{\mathrm{d}}=\frac{a \sin \Delta \Sigma}{i_{12}-\tan \Delta \Sigma} \text {. }
$$

综上，可以总结出一般化的包络环面蜗杆角修 形条件为.

i) 刀座轴线与蜗杆轴线正向夹角为 $90^{\circ}+\Delta \Sigma$, 蜗 杆毛坏与刀座间的传动比为 $i_{1 \mathrm{~d}} . \Delta \Sigma$ 和 $i_{1 \mathrm{~d}}$ 不相互独立, 二者之一确定后, 另一个由(16)式决定.

ii) 加工蜗杆的工艺中心距为 $a_{\mathrm{d}}=a \cos ^{2} \Delta \Sigma$.

iii) 如果产形面的单位法向量与刀座轴线垂直, $p_{\mathrm{d}}=0$, 刀座可以不延自身轴线移动, 只作旋转运动; 否则, 刀座须作螺旋运动, 螺旋运动的参数 $p_{\mathrm{d}}$ 由(17) 式决定.

角修形条件下, (15)式中 $A$ 和 $B$ 的值分别为

$$
A=\frac{T_{z}}{i_{\mathrm{ld}}}+p_{\mathrm{d}} n_{z}+\frac{a}{i_{12}} n_{1 z},
$$

$$
B=\frac{1}{i_{12}}\left[T_{x} \cos \left(\varphi / i_{1 \mathrm{~d}}\right)-T_{y} \sin \left(\varphi / i_{\mathrm{ld}}\right)-n_{o y} p_{\mathrm{d}} \varphi\right] .
$$

\section{3 一次包络的啮合特性参数}

\section{1 一次包络的两类界限曲线}

根据定义, 一次包络啮合函数 $\Phi_{\mathrm{d}}$ 对 $\varphi$ 求一阶偏 
导数, 可以得到一次包络的啮合界线函数 $\Phi_{\mathrm{d} \varphi}$. 令 $\Phi_{\mathrm{d}}=\Phi_{\mathrm{d} \varphi}=0$, 与(4)式联立, 得到砂轮产形面 $\Sigma_{\mathrm{d}}$ 上的一 次包络啮合界线 $L_{2}^{(1)}$ 的方程为

$$
\left\{\begin{array}{l}
\left(\boldsymbol{r}_{\mathrm{a}}\right)_{\mathrm{a}}=x_{\mathrm{a}} \boldsymbol{i}_{\mathrm{a}}+y_{\mathrm{a}} \boldsymbol{j}_{\mathrm{a}}+z_{\mathrm{a}} \boldsymbol{k}_{\mathrm{a}}, \\
\Phi_{\mathrm{d}}(\phi, \theta, \varphi)=0, \\
\Phi_{\mathrm{d} \varphi}(\phi, \theta, \varphi)=-i_{12} B \cos \Delta \Sigma / i_{1 \mathrm{~d}}=0 .
\end{array}\right.
$$

根据齿轮啮合理论, 可以得到一次包络曲率干 涉界线函数为

$$
\begin{aligned}
\Psi_{\mathrm{d}} & =\Psi_{\mathrm{d}}(\phi, \theta, \varphi) \\
& =\lambda_{\mathrm{d}}\left(\boldsymbol{g}_{1}\right)_{o \mathrm{~d}} \cdot\left(\boldsymbol{V}_{\mathrm{d} 1}\right)_{o \mathrm{~d}}+\mu_{\mathrm{d}}\left(\boldsymbol{g}_{2}\right)_{o \mathrm{~d}} \cdot\left(\boldsymbol{V}_{\mathrm{d} 1}\right)_{o \mathrm{~d}}+\Phi_{\mathrm{d} \varphi},
\end{aligned}
$$

式中,

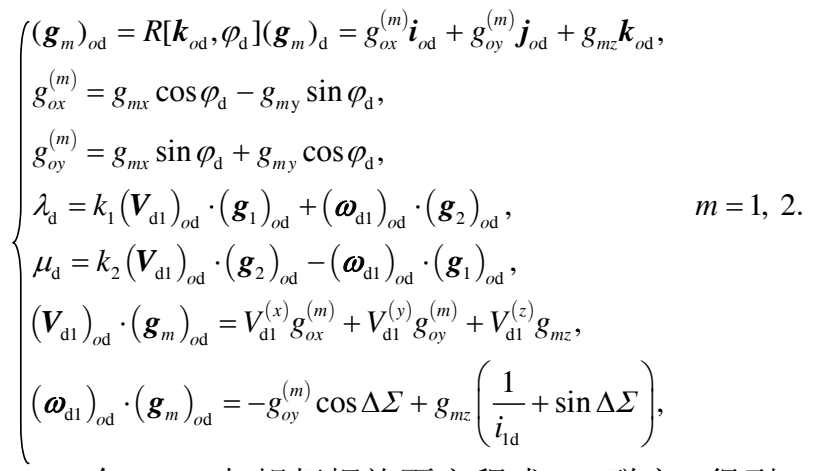

令 $\Psi_{\mathrm{d}}=0$ 与蜗杆螺旋面方程式(11)联立, 得到一 次包络曲率干涉界线 $L_{1}^{(1)}$ 的方程为

$$
\left\{\begin{array}{l}
\left(\boldsymbol{r}_{1}\right)_{1}=x_{o 1} \boldsymbol{e}_{1}(-\varphi)+y_{o 1} \boldsymbol{g}_{1}(-\varphi)+z_{1} \boldsymbol{k}_{1}, \\
\Phi_{\mathrm{d}}=0 \\
\Psi_{\mathrm{d}}=0
\end{array}\right.
$$

\section{2 蜗杆螺旋面的曲率参数}

根据齿轮啮合理论, 可以得到蜗杆螺旋面 $\Sigma_{1}$ 上 任意点沿 $\left(\boldsymbol{g}_{1}\right)_{\mathrm{od}}$ 和 $\left(\boldsymbol{g}_{2}\right)_{\mathrm{od}}$ 方向的法曲率分别为 $k_{\xi}^{(1)}=k_{1}-\lambda_{\mathrm{d}}^{2} / \Psi_{\mathrm{d}}$ 和 $k_{\eta}^{(1)}=k_{2}-\mu_{\mathrm{d}}^{2} / \Psi_{\mathrm{d}}$; 还可以得到沿 $\left(\boldsymbol{g}_{1}\right)_{o \mathrm{~d}}$ 方向的短程挠率为 $\tau_{\xi}^{(1)}=-\lambda_{\mathrm{d}} \mu_{\mathrm{d}} / \Psi_{\mathrm{d}}$.

在此基础上，能够得到蜗杆螺旋面 $\Sigma_{1}$ 上任意点 Gauss 曲率为 $K^{(1)}=k_{\xi}^{(1)} k_{\eta}^{(1)}-\left(\tau_{\xi}^{(1)}\right)^{2}$. 角修正型双圆环 面包络环面蜗杆螺旋面, 与标准型双圆环面包络环 面蜗杆螺旋面一样, 都是单参数圆环面族的包络面. 算例研究表明, 角修正蜗杆螺旋面上任意点 Gauss 曲 率 $K^{(1)}<0$, 为双曲点. 因此, 角修正双圆环面包络环 面蜗杆螺旋面为不可展的曲纹曲面.

\section{4 二次包络的啮合特性分析}

\section{1 蜗轮齿面方程}

对(13)式进行坐标变换后, 与 $\Phi=0$ 联立, 在坐标 系 $\sigma_{2}$ 中, 得到蜗轮齿面共轭区 $\Sigma_{2}$ 的方程为

$$
\left\{\begin{array}{l}
\left(\boldsymbol{r}_{2}\right)_{2}=R\left[\boldsymbol{k}_{2},-\varphi_{1} / i_{12}\right]\left\{R\left[\boldsymbol{i}_{o 2},-90^{\circ}\right]\left(\boldsymbol{r}_{1}^{*}\right)_{o 1}-a \boldsymbol{i}_{o 2}\right\} \\
\quad=x_{2} \boldsymbol{i}_{2}+y_{2} \mathbf{j}_{2}-y_{o 1}^{*} \boldsymbol{k}_{2}, \\
\Phi_{\mathrm{d}}=0 \\
\Phi=0,
\end{array}\right.
$$

式中 $x_{2}=\left(x_{o 1}^{*}-a\right) \cos \left(\varphi_{1} / i_{12}\right)+z_{1} \sin \left(\varphi_{1} / i_{12}\right), y_{2}=-\left(x_{o 1}^{*}-\right.$ a) $\sin \left(\varphi_{1} / i_{12}\right)+z_{1} \cos \left(\varphi_{1} / i_{12}\right), R\left[\boldsymbol{i}_{o 2},-90^{\circ}\right]$ 和 $R\left[\boldsymbol{k}_{2},-\varphi_{1} /\right.$ $i_{12}$ ] 分别为绕 $\boldsymbol{i}_{o 2}$ 轴和 $\boldsymbol{k}_{2}$ 轴的旋转变换矩阵.

由(15)式可见，与标准传动类似，角修形条件下， 二次包络啮合函数 $\Phi$ 仍可以分解为两个因子的乘积, 相应地, 蜗轮齿面共轭区 $\Sigma_{2}$ 也就由 $\Sigma_{2 A}$ 和 $\Sigma_{2 B}$ 两部分构 成. $\Sigma_{2 A}$ 和 $\Sigma_{2 B}$ 的方程分别为

$$
\begin{gathered}
\left\{\begin{array}{l}
\left(\boldsymbol{r}_{2}\right)_{2}=x_{2} \boldsymbol{i}_{2}+y_{2} \boldsymbol{j}_{2}-y_{o 1}^{*} \boldsymbol{k}_{2}, \\
\Phi_{\mathrm{d}}=0, \\
\varphi_{1}-\varphi=0,
\end{array}\right. \\
\left\{\begin{array}{l}
\left(\boldsymbol{r}_{2}\right)_{2}=x_{2} \boldsymbol{i}_{2}+y_{2} \boldsymbol{j}_{2}-y_{o 1}^{*} \boldsymbol{k}_{2}, \\
\Phi_{\mathrm{d}}=0, \\
A \sin \frac{\varphi_{1}-\varphi}{2}+B \cos \frac{\varphi_{1}-\varphi}{2}=0 .
\end{array}\right.
\end{gathered}
$$

由(22)式可见, 在二次包络过程中, 蜗杆螺旋面 $\Sigma_{1}$ 作为产形面, 在蜗轮齿面上范成出共轭区 $\Sigma_{2}$. 当 $\varphi_{1}=\varphi$ 时, 得到 $\Sigma_{2 A}$; 当 $\varphi_{1} \neq \varphi$ 时, 得到 $\Sigma_{2 B}$. 与标准传 动不同, 对于角修形传动, 因为在一次包络中 $\Sigma_{\mathrm{d}}$ 与 $\Sigma_{1}$ 的相对位置及相对运动和二次包络中 $\Sigma_{2 A}$ 与 $\Sigma_{1}$ 的相对 位置及相对运动不同, 所以 $\Sigma_{2 A}$ 不再是产形母圆环面 $\Sigma_{\mathrm{d}}$ 在蜗轮齿面上的再现. 但是, 因为 $\Sigma_{2 A}$ 毕竟是 $\varphi_{1}=\varphi$ 时得到的, 所以把 $\Sigma_{2 A}$ 称作名义原接触区. $\Sigma_{2 B}$ 是在二 次包络过程中形成的, 因此称为新接触区.

令 $\varphi_{1}$ 取不同值, 利用(22)式, 可以得到 $\Sigma_{2}$ 上、分 别位于 $\Sigma_{2 A}$ 和 $\Sigma_{2 B}$ 内的两族瞬时接触线, 这两族瞬时接 触线, 是 $\Sigma_{2 A}$ 和 $\Sigma_{2 B}$ 各自的特征线. 因此, 角修正双圆 环面二包传动也能实现瞬时双线接触, 这对于提高 传动副的承载能力是非常有利的.

由(11)与(22a)式可知, 共轭齿面偶 $\left[\Sigma_{1}, \Sigma_{2 A}\right]$ 在 $\Sigma_{1}$ 上的名义原瞬时接触线与共轭齿面偶 $\left[\Sigma_{\mathrm{d}}, \Sigma_{1}\right]$ 在 $\Sigma_{1}$ 上 
的瞬时接触线是相同的.

$\Sigma_{2 A}$ 和 $\Sigma_{2 B}$ 光滑相连, 交界线处存在公法线. 由 (22)式，可以得到它们的交界线方程为

$$
\left\{\begin{array}{l}
\left(\boldsymbol{r}_{2}\right)_{2}=x_{2} \boldsymbol{i}_{2}+y_{2} \boldsymbol{j}_{2}-y_{o 1}^{*} \boldsymbol{k}_{2}, \\
\Phi_{\mathrm{d}}(\phi, \theta, \varphi)=0, \\
\varphi_{1}-\varphi=0, \\
B(\phi, \theta, \varphi)=0 .
\end{array}\right.
$$

对比(18)与(23)式, 可以发现 $\Sigma_{2 A}$ 和 $\Sigma_{2 B}$ 的交界线 在蜗杆螺旋面 $\Sigma_{1}$ 上的共轭线, 是一次包络啮合界线 $L_{2}^{(1)}$ 的共轭线.

\section{2 二次包络的两类界线}

根据定义，由(15)式可以得到二次包络啮合界线 函数为

$$
\begin{aligned}
\Phi_{\varphi_{1}} & =\frac{\partial \Phi}{\partial \varphi_{1}}=\Phi_{\varphi_{1}}\left(\phi, \theta, \varphi, \varphi_{1}\right) \\
& =A \sin \left(\varphi_{1}-\varphi\right)+B \cos \left(\varphi_{1}-\varphi\right) .
\end{aligned}
$$

令 $\Phi=\Phi_{\varphi_{1}}=0$, 与(11)式联立, 得到二次包络啮 合界线曲线 $L_{2}^{(2)}$ 的方程为

$$
\left\{\begin{array}{l}
\left(\boldsymbol{r}_{1}\right)_{1}=x_{o 1} \boldsymbol{e}_{1}(-\varphi)+y_{o 1} \boldsymbol{g}_{1}(-\varphi)+z_{1} \boldsymbol{k}_{1}, \\
\Phi_{\mathrm{d}}(\phi, \theta, \varphi)=0, \\
\varphi_{1}-\varphi=0, \\
B(\phi, \theta, \varphi)=0 .
\end{array}\right.
$$

对比(18)与(25)式，可知蜗杆螺旋面 $\Sigma_{1}$ 上的二次 包络啮合界线 $L_{2}^{(2)}$, 是一次包络啮合界线共轭线, 所 以二次包络名义原接触线与 $L_{2}^{(2)}$ 相交于二次包络的 二类界点. 二次包络啮合界线 $L_{2}^{(2)}$ 是新接触线族的包 络线, 新接触线与 $L_{2}^{(2)}$ 相切于二次包络的二类界点. 因此, $L_{2}^{(2)}$ 上的点是 $\Sigma_{1}$ 上的名义原瞬时接触线与新接 触线的交点. 另外, 在蜗杆螺旋面 $\Sigma_{1}$ 上, $L_{2}^{(2)}$ 的一侧 存在双线接触区, $L_{2}^{(2)}$ 的位置决定了蜗杆双线工作长 度. 由(23)和(25)式还可知, 二次包络啮合界线 $L_{2}^{(2)}$ 的 共轭线, 是 $\Sigma_{2 A}$ 和 $\Sigma_{2 B}$ 的交界线.

根据齿轮啮合理论，二次包络瞬时接触线法向 量可以表示成

$$
(\boldsymbol{N})_{o 1}=N_{\xi}\left(\boldsymbol{\alpha}_{1}^{*}\right)_{o 1}+N_{\eta}\left(\boldsymbol{\alpha}_{2}^{*}\right)_{o 1},
$$

式中,

$$
\left\{\begin{aligned}
\left(\boldsymbol{\alpha}_{m}^{*}\right)_{o 1}= & R\left[\boldsymbol{k}_{o 1}, \varphi_{1}-\varphi\right] R\left[\boldsymbol{i}_{o \mathrm{~d}}, 90^{\circ}+\Delta \Sigma\right]\left(\boldsymbol{g}_{m}\right)_{o \mathrm{~d}} \\
& =\alpha_{m x} \boldsymbol{i}_{o 1}+\alpha_{m y} \boldsymbol{j}_{o 1}+\alpha_{m z} \boldsymbol{k}_{o 1}, \\
\alpha_{m x}= & g_{o x}^{(m)} \cos \left(\varphi_{1}-\varphi\right)+\left(g_{o y}^{(m)} \sin \Delta \Sigma+g_{m z} \cos \Delta \Sigma\right) \\
& \cdot \sin \left(\varphi_{1}-\varphi\right), \\
\alpha_{m y}= & g_{o x}^{(m)} \sin \left(\varphi_{1}-\varphi\right)-\left(g_{o y}^{(m)} \sin \Delta \Sigma+g_{m z} \cos \Delta \Sigma\right) \\
& \cdot \cos \left(\varphi_{1}-\varphi\right), \\
\alpha_{m z}= & g_{o y}^{(m)} \cos \Delta \Sigma-g_{m z} \sin \Delta \Sigma, \quad m=1,2 . \\
\left(\boldsymbol{V}_{12}\right)_{o 1} & \cdot\left(\boldsymbol{\alpha}_{m}^{*}\right)_{o 1}=-\alpha_{m x}\left(y_{o 1}^{*}-\frac{z_{o 1}}{i_{12}}\right)+\alpha_{m y} x_{o 1}^{*} \\
& -\alpha_{m z} \frac{x_{o 1}^{*}-a}{i_{12}}, \\
\left(\boldsymbol{\omega}_{12}\right)_{o 1} & \cdot\left(\boldsymbol{\alpha}_{m}^{*}\right)_{o 1}=\frac{\alpha_{m y}}{i_{12}}+\alpha_{m z}, \\
N_{\xi}= & k_{\xi}^{(1)}\left(\boldsymbol{V}_{12}\right)_{o 1} \cdot\left(\boldsymbol{\alpha}_{1}^{*}\right)_{o 1}+\tau_{\xi}^{(1)}\left(\boldsymbol{V}_{12}\right)_{o 1} \cdot\left(\boldsymbol{\alpha}_{2}^{*}\right)_{o 1} \\
& +\left(\boldsymbol{\omega}_{12}\right)_{o 1} \cdot\left(\boldsymbol{\alpha}_{2}^{*}\right)_{o 1}, \\
N_{\eta}= & \tau_{\xi}^{(1)}\left(\boldsymbol{V}_{12}\right)_{o 1} \cdot\left(\boldsymbol{\alpha}_{1}^{*}\right)_{o 1}+k_{\eta}^{(1)}\left(\boldsymbol{V}_{12}\right)_{o 1} \cdot\left(\boldsymbol{\alpha}_{2}^{*}\right)_{o 1} \\
& -\left(\boldsymbol{\omega}_{12}\right)_{o 1} \cdot\left(\boldsymbol{\alpha}_{1}^{*}\right)_{o 1},
\end{aligned}\right.
$$

根据(21)式, 可以认为共轭区 $\Sigma_{2}$ 的参数为 $\phi$ 和 $\varphi_{1}$. 由(15)式可以计算出 $\Phi$ 对 $\phi$ 的偏导数为

$$
\Phi_{\phi}=\frac{\partial \Phi}{\partial \phi}=\frac{\partial A}{\partial \phi}\left[1-\cos \left(\varphi_{1}-\varphi\right)\right]+\frac{\partial B}{\partial \phi} \sin \left(\varphi_{1}-\varphi\right) \text {. }
$$

由 (24) 和 (27) 式可见, 当 $\varphi_{1}=\varphi$ 且 $B=0$ 时, $\Phi_{\phi}=\Phi_{\varphi_{1}}=0$. 根据齿轮啮合理论, 此时 $(N)_{o 1}=0^{[11]}$. 因此, 二次包络的啮合界线 $L_{2}^{(2)}$ 及其共轭线, 是二次 包络瞬时接触线的奇点轨迹. 事实上, 因为二次包络 的啮合界线 $L_{2}^{(2)}$ 及其共轭线由瞬时接触线的交点构成, 所以这两条线上任意点瞬时接触线的法矢无定向.

根据齿轮啮合理论，还可以得到二次包络的曲 率干涉界线函数为 $\Psi=(N)_{o 1} \cdot\left(V_{12}\right)_{o 1}+\Phi_{\varphi_{1}}$. 当 $\Psi=0$ 时, 得到蜗轮齿面的曲率干涉界线 $L_{1}^{(2)}$. 由于共轭区 $\Sigma_{2}$ 分 为 $\Sigma_{2 A}$ 和 $\Sigma_{2 B}$ 两部分, 干涉界线 $L_{1}^{(2)}$ 也包含相应的两支. 与 $\Sigma_{2 A}$ 对应的一支, 是蜗轮齿面上瞬时接触线奇点轨 迹, 满足 $\Phi_{\varphi_{1}}=\Psi=0$, 所以不是 $\Sigma_{2 A}$ 上名义原接触线 族的包络线, 不引起 $\Sigma_{2 A}$ 根切. 与 $\Sigma_{2 B}$ 对应的一支, 如 果存在, 是二包新接触线族的包络线. 通过分析二包 新接触线的分布与走向可知, 与 $\Sigma_{2 B}$ 对应的曲率干涉 
界线一般不会在蜗轮齿面上出现. 综上所论, 角修正 双圆环面二包副的蜗轮齿面通常无根切.

\section{3 蜗杆副微观啮合特性}

通常, 以诱导主曲率半径系数 $k_{\rho}=\Psi /\left[a\left(N_{\xi}^{2}+\right.\right.$ $\left.\left.N_{\eta}^{2}\right)\right]$ 和 滑 动 角 $\theta_{v t}=\arcsin \left[\left|\Phi_{\varphi_{1}}-\Psi\right| /\left(\left|\left(\boldsymbol{V}_{12}\right)_{o 1}\right|\right.\right.$ $\sqrt{N_{\xi}^{2}+N_{\eta}^{2}}$ )], 作为衡量环面蜗杆副微观啮合质量的 标准 ${ }^{[10,13]}$.

此外, 还可得到共轭区 $\Sigma_{2}$ 的 Gauss 曲率为 $K^{(2)}=$ $\left(k_{\xi}^{(1)}-N_{\xi}^{2} / \Psi\right)\left(k_{\eta}^{(1)}-N_{\eta}^{2} / \Psi\right)-\left(\tau_{\xi}^{(1)}-N_{\xi} N_{\eta} / \Psi\right)^{2}$. 算例研 究表明, 名义原接触区 $\Sigma_{2 A}$ 由椭圆点和双曲点构成, 在 $\Sigma_{2 A}$ 靠近新接触区 $\Sigma_{2 B}$ 的一侧为双曲点, 另一侧为椭 圆点. 这就从曲率特征的角度, 反映了名义原接触区 $\Sigma_{2 A}$ 与蜗杆产形母圆环面 $\Sigma_{\mathrm{d}}$ 的差异.

\section{5 角修形传动的啮合特性}

\section{1 角修形的物理意义}

(14)式中令 $\varphi_{1}=\varphi$, 可以得到共轭齿面偶 $\left[\Sigma_{1}, \Sigma_{2 A}\right]$ 的相对运动角速度矢和相对运动速度矢, 在坐标系 $\sigma_{o \mathrm{~d}}$ 中, 它们与 $\boldsymbol{\omega}_{\mathrm{d} 1}$ 和 $\boldsymbol{V}_{\mathrm{d} 1}$ 的关系分别为

$$
\left\{\begin{array}{l}
\left(\omega_{12}^{(A)}\right)_{o \mathrm{~d}}=-\frac{i_{1 \mathrm{~d}}}{i_{12} \cos \Delta \Sigma}\left(\boldsymbol{\omega}_{\mathrm{d} 1}\right)_{o \mathrm{~d}}, \\
\left(\boldsymbol{V}_{12}^{(A)}\right)_{o \mathrm{~d}}=-\frac{i_{1 \mathrm{~d}}}{i_{12} \cos \Delta \Sigma}\left(\boldsymbol{V}_{\mathrm{d} 1}\right)_{o \mathrm{~d}} .
\end{array}\right.
$$

由(28)式可见, 共轭齿面偶 $\left[\Sigma_{\mathrm{d}}, \Sigma_{1}\right]$ 与 $\left[\Sigma_{1}, \Sigma_{2 \mathrm{~A}}\right]$ 的 瞬时相对螺旋运动轴共线, 它们的相对运动速度反向 共线, 且相对运动速度数值之比与相对运动角速度数 值之比相等, 比值为角修形参数的函数. 对于标准传 动, 以上结论仍然成立, 只不过 $i_{1 \mathrm{~d}}=i_{12}$ 且 $\Delta \Sigma=0$.

\section{2 瞬时接触线奇点轨迹的切方向}

在蜗轮齿面 $\Sigma_{2}$ 上的瞬时接触线奇点轨迹上任取 一点 $P\left(x_{P}, y_{P}, z_{P}\right), \sigma_{2}$ 中点 $P$ 的径矢为 $\left(\boldsymbol{r}_{P}\right)_{2}$, 与点 $P$ 相对应的蜗杆转角为 $\varphi_{1}^{(P)}$, 点 $P$ 处存在蜗轮齿面 $\Sigma_{2}$ 的 法线 $\left(\boldsymbol{n}_{P}\right)_{2}$.

以 $\varphi$ 为蜗轮齿面瞬时接触线奇点轨迹的参数, 利用相对微分法 ${ }^{[5]}$, 得到蜗轮齿面瞬时接触线奇点轨 迹的切向量为

$$
\frac{\mathrm{d}_{2} \boldsymbol{r}_{2}}{\mathrm{~d} \varphi}=\frac{\mathrm{d} \phi}{\mathrm{d} \varphi} \frac{\partial_{\mathrm{d}} \boldsymbol{r}_{\mathrm{d}}}{\partial \phi}+\frac{\mathrm{d} \theta}{\mathrm{d} \varphi} \frac{\partial_{\mathrm{d}} \boldsymbol{r}_{\mathrm{d}}}{\partial \theta}+3\left(\boldsymbol{V}_{\mathrm{d} 1}+\boldsymbol{V}_{12}\right) .
$$

蜗轮齿面瞬时接触线奇点轨迹方程式(23)中, $\Phi_{\mathrm{d}}=0$ 与 $B=0$ 分别对 $\varphi$ 求偏导数, 可以得到

$$
\left\{\begin{array}{l}
\Phi_{\mathrm{d} \phi} \frac{\mathrm{d} \phi}{\mathrm{d} \varphi}+\Phi_{\mathrm{d} \theta} \frac{\mathrm{d} \theta}{\mathrm{d} \varphi}=0, \\
B_{\phi} \frac{\mathrm{d} \phi}{\mathrm{d} \varphi}+B_{\theta} \frac{\mathrm{d} \theta}{\mathrm{d} \varphi}+B_{\varphi}=0,
\end{array}\right.
$$

式中,

$$
\left\{\begin{aligned}
\Phi_{\mathrm{d} \phi} & =-\rho \lambda_{\mathrm{d}} \\
\Phi_{\mathrm{d} \theta} & =-\mu_{\mathrm{d}}(\rho \sin \phi+p), \\
B_{\varphi}= & \frac{-1}{i_{12} i_{\mathrm{ld}}}\left(T_{x} \sin \frac{\varphi}{i_{\mathrm{ld}}}+T_{y} \cos \frac{\varphi}{i_{\mathrm{ld}}}+p_{\mathrm{d}} n_{o x} \varphi+i_{\mathrm{ld}} p_{\mathrm{d}} n_{o y}\right), \\
B_{\phi}= & \frac{-1}{i_{12}}\left\{\sin \theta(p \sin \phi+q \cos \phi) \cos \frac{\varphi}{i_{\mathrm{ld}}}\right. \\
& +\left[\sin \beta \cos \theta(p \sin \phi+q \cos \phi)+a_{0} g_{1 z}\right] \sin \frac{\varphi}{i_{\mathrm{ld}}} \\
& \left.+p_{\mathrm{d}} \varphi g_{o y}^{(1)}\right\}, \\
B_{\theta}= & \frac{1}{i_{12}}\left\{\left[\cos \theta(p \cos \phi-q \sin \phi)-p_{\mathrm{d}} n_{x} \varphi \sin \beta\right] \cos \frac{\varphi}{i_{\mathrm{ld}}}\right. \\
& +\left[p_{\mathrm{d}} \varphi \sin \phi \sin \theta-\sin \beta \sin \theta(p \cos \phi-q \sin \phi)\right. \\
& \left.\left.+a_{0} n_{x} \cos \beta\right] \sin \frac{\varphi}{i_{\mathrm{ld}}}\right\} .
\end{aligned}\right.
$$

解方程组(30)得到

$$
\left\{\begin{array}{l}
\frac{\mathrm{d} \phi}{\mathrm{d} \varphi}=\frac{\mu_{\mathrm{d}} B_{\varphi}(\rho \sin \phi+p)}{\left|\begin{array}{ll}
\rho \lambda_{\mathrm{d}} & \mu_{\mathrm{d}}(\rho \sin \phi+p) \\
B_{\phi} & B_{\theta}
\end{array}\right|}, \\
\frac{\mathrm{d} \theta}{\mathrm{d} \varphi}=\frac{-\rho \lambda_{\mathrm{d}} B_{\varphi}}{\left|\begin{array}{cc}
\rho \lambda_{\mathrm{d}} & \mu_{\mathrm{d}}(\rho \sin \phi+p) \\
B_{\phi} & B_{\theta}
\end{array}\right|} .
\end{array}\right.
$$

记 $(\boldsymbol{J})_{\mathrm{d}}=\frac{\mathrm{d} \phi}{\mathrm{d} \varphi} \frac{\partial_{\mathrm{d}} \boldsymbol{r}_{\mathrm{d}}}{\partial \phi}+\frac{\mathrm{d} \theta}{\mathrm{d} \varphi} \frac{\partial_{\mathrm{d}} \boldsymbol{r}_{\mathrm{d}}}{\partial \theta}=J_{x} \boldsymbol{i}_{\mathrm{d}}+J_{y} \boldsymbol{j}_{\mathrm{d}}+J_{z} \boldsymbol{k}_{\mathrm{d}}$, 则 $J_{x}=$ $\rho g_{1 x} \frac{\mathrm{d} \phi}{\mathrm{d} \varphi}-y_{\mathrm{a}} \frac{\mathrm{d} \theta}{\mathrm{d} \varphi}, J_{y}=\rho g_{1 y} \frac{\mathrm{d} \phi}{\mathrm{d} \varphi}+x_{\mathrm{a}} \sin \beta \frac{\mathrm{d} \theta}{\mathrm{d} \varphi}, J_{z}=\rho g_{1 z} \frac{\mathrm{d} \phi}{\mathrm{d} \varphi}-$ $x_{\mathrm{a}} \cos \beta \frac{\mathrm{d} \theta}{\mathrm{d} \varphi}$. 于是由(29)式, 在坐标系 $\sigma_{o 1}$ 中, 得到蜗 
轮齿面瞬时接触线奇点轨迹的切向量为

$$
\begin{aligned}
(\ell)_{o 1}= & R\left[\boldsymbol{i}_{o 1}, 90^{\circ}+\Delta \Sigma\right]\left[R\left[\boldsymbol{k}_{o \mathrm{~d}}, \varphi_{\mathrm{d}}\right](\boldsymbol{J})_{\mathrm{d}}\right. \\
& \left.+3\left(\boldsymbol{V}_{\mathrm{d} 1}\right)_{o \mathrm{~d}}\right]+3\left(\boldsymbol{V}_{12}\right)_{o 1} \\
= & \ell_{x} \boldsymbol{i}_{o 1}+\ell_{y} \boldsymbol{j}_{o 1}+\ell_{z} \boldsymbol{k}_{o 1},
\end{aligned}
$$

式中,

$$
\left\{\begin{aligned}
\ell_{x}= & J_{x} \cos \frac{\varphi}{i_{\mathrm{ld}}}-J_{y} \sin \frac{\varphi}{i_{\mathrm{ld}}} \\
& -\frac{3 \sin \Delta \Sigma}{i_{12}}\left[\frac{y_{o \mathrm{~d}}}{i_{\mathrm{ld}}}\left(i_{12} \sin \Delta \Sigma+\cos \Delta \Sigma\right)+z_{\mathrm{d}}+p_{\mathrm{d}} \varphi\right], \\
\ell_{y}= & -\left(J_{x} \sin \frac{\varphi}{i_{\mathrm{ld}}}+J_{y} \cos \frac{\varphi}{i_{\mathrm{ld}}}\right) \sin \Delta \Sigma-J_{z} \cos \Delta \Sigma \\
& -\frac{3 \sin \Delta \Sigma}{i_{\mathrm{ld}}}\left(x_{o \mathrm{~d}}+a \cos \Delta \Sigma\right), \\
\ell_{z}= & \left(J_{x} \sin \frac{\varphi}{i_{\mathrm{ld}}}+J_{y} \cos \frac{\varphi}{i_{\mathrm{ld}}}\right) \cos \Delta \Sigma-J_{z} \sin \Delta \Sigma \\
& +\frac{3 \sin \Delta \Sigma}{i_{12} i_{\mathrm{ld}}}\left(x_{o \mathrm{~d}}-a \sin \Delta \Sigma\right) .
\end{aligned}\right.
$$

所以, 在坐标系 $\sigma_{2}$ 中可以得到单位化后的蜗轮 齿面瞬时接触线奇点轨迹的切向量为

$$
\begin{aligned}
(\boldsymbol{L})_{2}= & \frac{\ell_{x} \cos \left(\varphi_{1} / i_{12}\right)+\ell_{z} \sin \left(\varphi_{1} / i_{12}\right)}{\sqrt{\ell_{x}^{2}+\ell_{y}^{2}+\ell_{z}^{2}}} \boldsymbol{i}_{2} \\
& -\frac{\ell_{x} \sin \left(\varphi_{1} / i_{12}\right)-\ell_{z} \cos \left(\varphi_{1} / i_{12}\right)}{\sqrt{\ell_{x}^{2}+\ell_{y}^{2}+\ell_{z}^{2}}} \boldsymbol{j}_{2} \\
& -\frac{\ell_{y}}{\sqrt{\ell_{x}^{2}+\ell_{y}^{2}+\ell_{z}^{2}}} \boldsymbol{k}_{2} .
\end{aligned}
$$

在给定点 $P$, 蜗轮齿面瞬时接触线奇点轨迹的切 向量 $\left(\boldsymbol{L}_{P}\right)_{2}$ 为已知向量.

\section{3 蜗杆螺旋面和蜗轮齿面的法截线}

在点 $P$ 以蜗轮齿面瞬时接触线奇点轨迹的法平 面作共轭齿面偶 $\left[\Sigma_{1}, \Sigma_{2}\right]$ 的法截面 $\Pi$, 如图 5 所示, 则 平面 $\Pi$ 的单位法向量为 $L_{P}$. 于是可以得到蜗杆螺旋 面 $\Sigma_{1}$ 在法截面 $\Pi$ 内的法截线 $\Gamma_{w}$ 的方程为

$$
\left\{\begin{array}{l}
\left(\boldsymbol{r}_{\mathrm{w}}\right)_{2}=x_{2}\left(\phi, \theta, \varphi, \varphi_{1}^{(P)}\right) \boldsymbol{i}_{2}+y_{2}\left(\phi, \theta, \varphi, \varphi_{1}^{(P)}\right) \boldsymbol{j}_{2} \\
\quad-y_{o 1}^{*}\left(\phi, \theta, \varphi, \varphi_{1}^{(P)}\right) \boldsymbol{k}_{2}, \\
\Phi_{\mathrm{d}}=0, \\
\left(\boldsymbol{L}_{P}\right)_{2} \cdot\left[\left(\boldsymbol{r}_{w}\right)_{2}-\left(\boldsymbol{r}_{p}\right)_{2}\right]=0 .
\end{array}\right.
$$

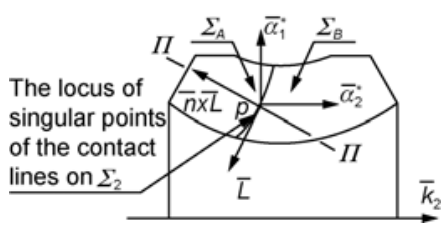

图 5 蜗轮齿面瞬时接触线奇点处的法截面

同理, 还可以得到蜗轮齿面名义原接触区 $\Sigma_{2 A}$ 和 新接触区 $\Sigma_{2 B}$ 在法截面 $\Pi$ 内的法截线 $\Gamma_{A}$ 与 $\Gamma_{B}$ 的方程分

\begin{tabular}{|c|c|c|c|c|c|}
\hline & 参数及单位 & 甲 & 乙 & 丙 & 丁 \\
\hline \multirow{3}{*}{ 设计参数 } & $a(\mathrm{~mm})$ & 240 & 200 & 320 & 200 \\
\hline & $i_{12}$ & 40 & 12 & 4 & 12 \\
\hline & 蜗杆头数 $Z_{1}$ & 1 & 3 & 12 & 3 \\
\hline \multirow{3}{*}{ 工艺参数 } & $r_{\mathrm{d}}(\mathrm{mm})$ & 100 & 120 & 95 & 120 \\
\hline & $\rho=5.5 m_{\mathrm{t}}(\mathrm{mm})$ & 52.11 & 47.97 & 58.44 & 47.97 \\
\hline & $\beta\left(^{\circ}\right)$ & 8 & 8 & 25 & 23 \\
\hline \multirow{4}{*}{ 修形参数 } & $\Delta \Sigma\left(^{\circ}\right)$ & 11.4821 & 14.02 & 20 & 0 \\
\hline & $i_{1 \mathrm{~d}}$ & 39 & 11.4 & 3.42 & 0 \\
\hline & $\Delta a(\mathrm{~mm})$ & -9.5145 & -11.74 & -37.43 & 0 \\
\hline & $p_{\mathrm{d}}(\mathrm{mm})$ & 1.2007 & 4.1247 & 30.10 & 0 \\
\hline \multirow{2}{*}{$\begin{array}{l}\text { 传动副特 } \\
\text { 性参数 }\end{array}$} & $\begin{array}{c}\text { 环面蜗杆边齿顶厚 } \\
\text { 系数 } k_{\mathrm{s}}\end{array}$ & 0.36 & 0.38 & 0.36 & 0.37 \\
\hline & $\begin{array}{c}\text { 蜗杆名义工作长度 } \\
\text { 系数 } k_{\mathrm{L}}\end{array}$ & 0.99 & 0.74 & 0.54 & 0.81 \\
\hline
\end{tabular}
别为

$$
\begin{gathered}
\left\{\begin{array}{l}
\left(\boldsymbol{r}_{\mathrm{g}}\right)_{2}=x_{2} \boldsymbol{i}_{2}+y_{2} \boldsymbol{j}_{2}-y_{o 1}^{*} \boldsymbol{k}_{2}, \\
\Phi_{\mathrm{d}}(\phi, \theta, \varphi)=0, \\
\varphi_{1}-\varphi=0, \\
\left(\boldsymbol{L}_{P}\right)_{2} \cdot\left[\left(\boldsymbol{r}_{\mathrm{g}}\right)_{2}-\left(\boldsymbol{r}_{p}\right)_{2}\right]=0,
\end{array}\right. \\
\left\{\begin{array}{l}
\left(\boldsymbol{r}_{\mathrm{g}}\right)_{2}=x_{2} \boldsymbol{i}_{2}+y_{2} \boldsymbol{j}_{2}-y_{o 1}^{*} \boldsymbol{k}_{2}, \\
\Phi_{\mathrm{d}}=0, \\
A \sin \frac{\varphi_{1}-\varphi}{2}+B \cos \frac{\varphi_{1}-\varphi}{2}=0, \\
\left(\boldsymbol{L}_{P}\right)_{2} \cdot\left[\left(\boldsymbol{r}_{\mathrm{g}}\right)_{2}-\left(\boldsymbol{r}_{p}\right)_{2}\right]=0 .
\end{array}\right.
\end{gathered}
$$

通过分析法截面 $\Pi$ 内的法截线 $\Gamma_{w}, \Gamma_{A}$ 和 $\Gamma_{B}$ 的位 置关系, 可以明确蜗杆螺旋面 $\Sigma_{1}$ 、蜗轮齿面名义原接 触区 $\Sigma_{2 A}$ 和新接触区 $\Sigma_{2 B}$ 的位置关系, 还可以计算传动 副瞬时接触线奇点周围蜗杆螺旋面与蜗轮齿面的法 向间隙.

\section{6 算例与讨论}

各算例的参数列于表 1 . 算例甲、乙和丙为角修

表 1 各算例的参数 
形传动; 为了进行角修形传动与标准传动的对比, 给 出标准传动算例丁, 其设计参数与算例乙相同, 工艺 参数相近.

由于头数少、传动比大, 算例甲和乙以工艺传动 比为基本角修形变量. 此时据(16)式, 应注意使得 $i_{1 \mathrm{~d}}<i_{12}$. 由于头数多、传动比小, 算例丙以 $\Delta \Sigma$ 为基本 角修形变量, 有利于避免蜗杆边齿顶变尖.

利用蜗杆螺旋面方程式(11), 可以计算出蜗杆轴 截面内边齿顶厚, 用该边齿顶厚与蜗轮端面模数 $m_{\mathrm{t}}$ 的比值 $k_{\mathrm{s}}$ 作为环面蜗杆边齿顶厚系数, 通常以 $k_{\mathrm{s}} \geqslant 0.35$ 为环面蜗杆边齿顶无变尖的判据 ${ }^{[10,13]}$. 表 1 的计算结果表明, 角修正双圆环面包络环面蜗杆, 在 头数多、传动比小的情况下, 能够有效地避免边齿顶 变尖.

以算例丙为例, 研究多头数、小传动比条件下蜗
杆的根切特性. 图 6 在蜗杆轴截面内及其产形砂轮轴 截面内, 分别绘出算例丙的一次包络曲率干涉界线 $L_{1}^{(1)}$ 及其共轭线, 并且在蜗杆螺旋面上任选一点 $M$, 计算了该点曲率干涉界线函数的值 $\Psi_{\mathrm{d}}^{(M)}<0$. 由该图 可见, 因为曲线 $L_{1}^{(1)}$ 的共轭线位于产形砂轮实体内部, 曲线 $L_{1}^{(1)}$ 上诸点不能参与实际工艺啮合, 故曲线 $L_{1}^{(1)}$ 不会进入蜗杆螺旋面 $\Sigma_{1}$; 于是蜗杆螺旋面 $\Sigma_{1}$ 整个位 于曲率干涉界线 $L_{1}^{(1)}$ 的 $\Psi_{\mathrm{d}}<0$ 一侧; 又因为产形圆环 面 $\Sigma_{\mathrm{d}}$ 的单位法向量是由砂轮实体指向空域, 所以算 例丙中蜗杆无根切.

图 7 和 8 用虚线分别绘出了算例乙和丁的接触区, 用实线绘出了此二算例的瞬时接触线. 为便于观察, 蜗杆螺旋面 $\Sigma_{1}$ 上的接触区在坐标系 $\left(o_{1} ; \boldsymbol{j}_{o 1}, \boldsymbol{k}_{o 1}\right)$ 中绘 制. 蜗轮齿面接触区为 $A B H G$, 其中 $A B F E$ 为名义原

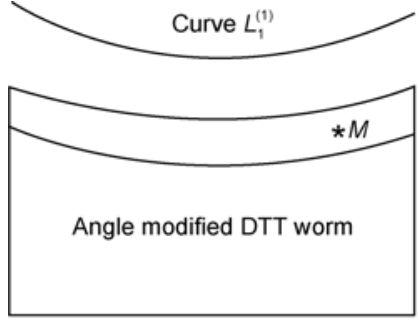

(a)

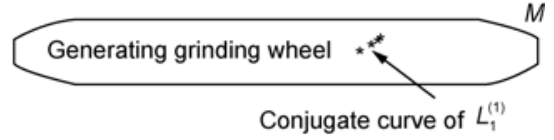

(b)

图 6 算例丙的 $\boldsymbol{L}_{1}^{(1)}$ 曲线(蜗杆轴截面内)(a)和曲线共轭线(砂轮轴截面内)(b)

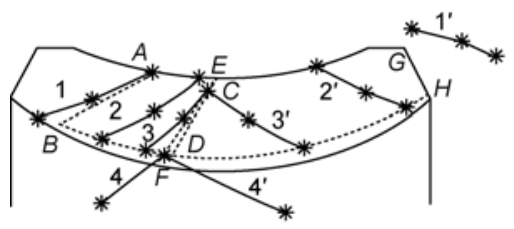

(a)

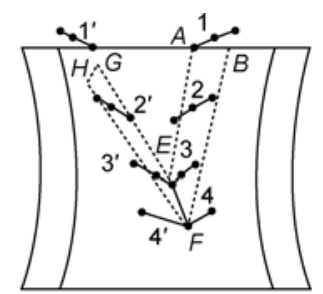

(b)

图 7 算例乙蜗轮齿面瞬时接触线族(a)和蜗杆瞬时接触线族(b)

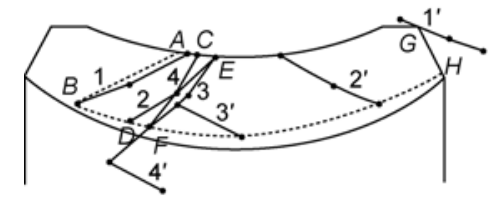

(a)

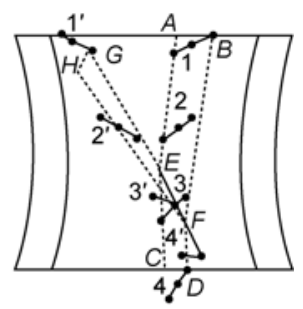

(b)

图 8 算例丁蜗轮齿面瞬时接触线族(a)和蜗杆瞬时接触线族(b) 
接触区 $\Sigma_{2 A}, E F H G$ 为新接触区 $\Sigma_{2 B} . A B$ 线与蜗杆啮入 端对应. $E F$ 线是 $\Sigma_{2 A}$ 与 $\Sigma_{2 B}$ 的交界线. $C D$ 线与蜗杆啮 出端相对应.

对于标准传动算例丁, 如图 8 所示, $C D$ 线在原接 触区内, $C D F E$ 构成蜗轮齿面上啮合质量差的二次接 触区. 通过选择足够的角修形量, 算例乙中 $C D$ 线能 够完全移入新接触区内, 所以二次接触区被切去, 如 图 7 所示. 不过, 角修形量也不能过大, 否则会过多 地减小名义原接触区, 使蜗杆啮入端瞬时接触线密 集, 传动副在较长的时间内处于单线接触状态; 而且 可能还会导致环面蜗杆边齿顶变尖.

在蜗杆螺旋面 $\Sigma_{1}$ 上, 名义原接触线族 $1,2, \cdots$ 与 $E F$ 线相交, 新接触线族 $1^{\prime}, 2^{\prime}, \cdots$ 被其包络. 对于标 准传动, 每条原接触线在一包二界共轭点一侧的部 分将在 $C D$ 线与 $E F$ 线之间实现与蜗轮齿面上二次接 触区的啮合. 对于角修形传动, 由于蜗轮齿面上 $C D$ 线移入了新接触区, 因此, 每条名义原接触线在一包 二界共轭点一侧的部分将不能在 $C D$ 线与 $E F$ 线之间 实现与蜗轮齿面啮合. 这就造成从啮出端到 $E F$ 线, 成为角修形蜗杆螺旋面 $\Sigma_{1}$ 上的无效区.

由此可见, 角修形蜗杆不可能利用其全长, 但是, 角修形蜗杆整个工作长度在理论上都是双线工作区. 标准蜗杆虽然可以利用其全长, 但是在全长范围内 存在单线工作区与双线工作区, 其中单线工作区和 蜗轮齿面上二次接触区相啮合.

有鉴于此, 可以采用啮入端 $A B$ 线到 $F$ 点的距离
与蜗杆螺旋部分长度 $L_{w}$ 的比值定义蜗杆名义工作长 度系数 $k_{\mathrm{L}}$, 作为评价角修形传动性能的指标. 传动副 具有较长的蜗杆名义工作长度, 能够延长瞬时接触 线总长, 对提高传动副承载能力、增厚弹流润滑油膜 都很有帮助. 由表 1 的计算结果可见, 角修正双圆环 面二包传动通常具有较长的蜗杆名义工作长度, 特 别是蜗杆头数少、传动比大时.

角修形蜗轮齿面上两族瞬时接触线大致成“八” 字形分布. 由于切去了二次接触区, 因此名义原接触 区 $\Sigma_{2 A}$ 内的名义原接触线族只保留了二包二界共轭点 一侧的部分, 于是名义原接触线族在 $\Sigma_{2 A}$ 中无交叉; 新接触区 $\Sigma_{2 B}$ 内的瞬时接触线族分布稀疏, 移动速度 快, 有利于传动副的承载与散热.

每一条名义原接触线和与之相对应的新接触线 相交于 $E F$ 线上一点. 分别以算例乙和丁原接触线 3 和新接触线 $3^{\prime}$ 在 $E F$ 线上的交点为 $P$ 点, 作法截面 $\Pi$, 得到蜗杆螺旋面 $\Sigma_{1}$ 与蜗轮齿面 $\Sigma_{2}$ 的法截线如图 9 所 示. 有关数值计算结果列于表 2 .

图 9 中以 $\left(\boldsymbol{n}_{P}\right)_{2} \times\left(\boldsymbol{L}_{P}\right)_{2}$ 为横坐标正向, $\left(\boldsymbol{n}_{P}\right)_{2}$ 为纵 坐标方正向, 点 $P$ 为坐标原点, 则横坐标为负的部分 对应新接触区 $\Sigma_{2 B}$, 横坐标为正的部分对应原接触区 或名义原接触区 $\Sigma_{2 A}$.

对于标准传动算例丁, 在蜗轮齿面 $\Sigma_{2}$ 上, $E F$ 线 是原接触线族的包络线，构成原接触区的自然边界. 因此, 图 9(b)中原接触区 $\Sigma_{2 A}$ 的法截线 $\Gamma_{A}$, 在新接触 区 $\Sigma_{2 B}$ 对应的横轴负半轴部分不出现, 蜗杆螺旋面 $\Sigma_{1}$

表 2 算例乙和丁法截线上对应点的法向距离

\begin{tabular}{|c|c|c|c|c|}
\hline 算例 & $\begin{array}{c}\text { 第(2)点处 } \Sigma_{1} \text { 与 } \Sigma_{2 A} \text { 的距离 } \\
d_{1 A}^{(2)}(\mathrm{mm})\end{array}$ & $\begin{array}{c}\text { 第(2)点处 } \Sigma_{1} \text { 与 } \Sigma_{2 B} \text { 的距离 } \\
d_{1 B}^{(2)}(\mathrm{mm})\end{array}$ & $\begin{array}{c}\text { 第(4)点处 } \Sigma_{1} \text { 与 } \Sigma_{2 A} \text { 的距离 } \\
d_{1 A}^{(4)}(\mathrm{mm})\end{array}$ & $\begin{array}{c}\text { 第(4)点处 } \Sigma_{1} \text { 与 } \Sigma_{2 B} \text { 的距离 } \\
d_{1 B}^{(4)}(\mathrm{mm})\end{array}$ \\
\hline 乙 & -0.6721 & 0.0030 & 0.1472 & -0.0031 \\
\hline 丁 & - & 0.0004 & 0.3182 & -0.0002 \\
\hline
\end{tabular}
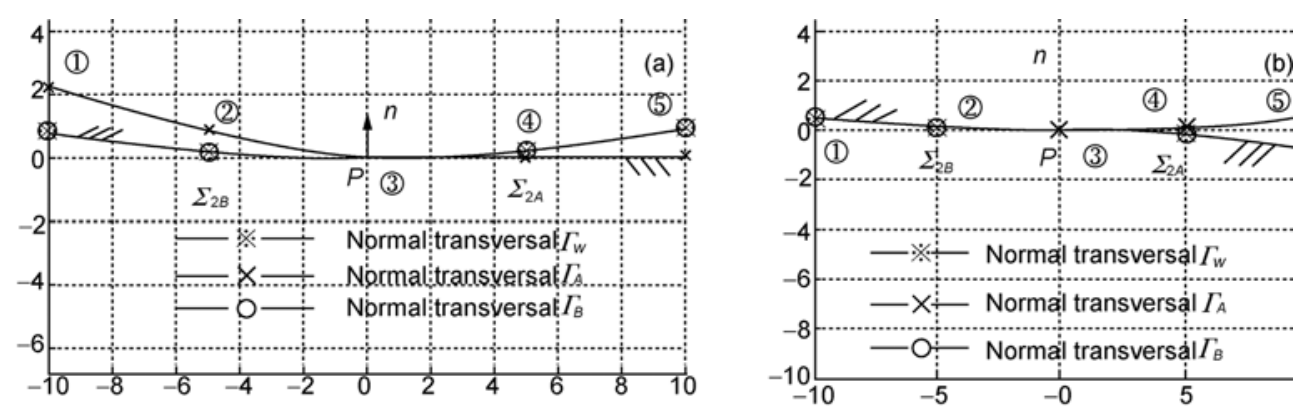

图 9 算例乙的法截线(a)和算例丁的法截线 $(\mathbf{b})$ 
也就与原接触区 $\Sigma_{2 A}$ 在点 $P$ 相切而不密切. 由表 2 的 计算结果可见, 新接触区 $\Sigma_{2 B}$ 的法截线 $\Gamma_{B}$ 与蜗杆螺旋 面 $\Sigma_{1}$ 的法截线 $\Gamma_{w}$ 在点 $P$ 相交叉, 在原接触区 $\Sigma_{2 A}$ 内进 入蜗杆实体, 所以新接触区 $\Sigma_{2 B}$ 与蜗杆螺旋面 $\Sigma_{1}$ 沿 $E F$ 线密切.

对于角修形传动算例乙，作为 $\Sigma_{2 A}$ 特征线的名义 原接触线族, 不再是一次包络瞬时接触线族在蜗轮 齿面 $\Sigma_{2}$ 上的再现. 虽然 $\Sigma_{2}$ 上 $E F$ 线仍满足 $\Phi_{\mathrm{d} \varphi}=0$, 但 $E F$ 线也不再是名义原接触线族的包络线, 名义原接 触线与之相交. 如图 9(a)所示, 名义原接触区 $\Sigma_{2 A}$ 的 法截线 $\Gamma_{A}$ 与蜗杆螺旋面 $\Sigma_{1}$ 的法截线 $\Gamma_{w}$ 在点 $P$ 相交叉, 在新接触区 $\Sigma_{2 B}$ 内进入蜗杆实体, 说明名义原接触区 $\Sigma_{2 A}$ 与蜗杆螺旋面 $\Sigma_{1}$ 沿 $E F$ 线密切. 由表 2 的计算结果 说明, 新接触区 $\Sigma_{2 B}$ 的法截线 $\Gamma_{B}$ 与蜗杆螺旋面 $\Sigma_{1}$ 的法 截线 $\Gamma_{w}$ 在点 $P$ 相交叉, 在名义原接触区 $\Sigma_{2 A}$ 内进入蜗 杆实体, 表明新接触区 $\Sigma_{2 B}$ 与蜗杆螺旋面 $\Sigma_{1}$ 沿 $E F$ 线密 切.

各瞬时接触线由蜗轮齿顶到齿根依次取 $a, b$ 和 $c$ 三点, 分别计算算例乙和丁在各点的微观啮合质量 参数 $k_{\rho}$ 和 $\theta_{v t}$ 的值, 结果列于表 3 和 4 . 角修形传动与 标准传动一样, 瞬时接触线奇点轨迹 $E F$ 线上诸点 $k_{\rho} \rightarrow \infty$, 滑动角 $\theta_{v t}$ 无定义.

由于角修形使得蜗杆螺旋面 $\Sigma_{1}$ 与 $\Sigma_{2 A}$ 和 $\Sigma_{2 B}$ 都密 切, 因此, 相对于标准传动, 角修形传动蜗杆螺旋面 $\Sigma_{1}$ 与 $\Sigma_{2 A}$ 的微观啮合质量得到了改善, 表 3 和 4 从 $k_{\rho}$ 的数值上反映了这一点. 不过, 蜗杆螺旋面 $\Sigma_{1}$ 与 $\Sigma_{2 A}$
和 $\Sigma_{2 B}$ 密切的程度不同, $\Sigma_{1}$ 与 $\Sigma_{2 B}$ 的贴近程度, 远远超 过它和 $\Sigma_{2 A}$ 的贴近程度, 所以角修形蜗轮齿面新接触 区的微观啮合质量仍好于名义原接触区. 此外, 角修 形对蜗杆副滑动角影响不大, 对于新接触区 $\Sigma_{2 B}$ 的微 观啮合质量一般影响也不大.

总的看来, 角修形能够使得整个蜗轮齿面的微 观啮合质量得到改善. 角修正双圆环面二包传动的 微观啮合质量稳定, 随着蜗杆头数增加、传动比减小 变化不大.

\section{7 结论}

本文主要做了两个方面的工作，一是丰富和发 展了角修形传动啮合理论; 一是基于该理论提出了 一种新型环面蜗杆传动一一角修正双圆环面二次包络 环面蜗杆传动.

角修形传动啮合理论方面, 本文得到的主要结 论为.

1) 得到了一般化的角修形条件, 解释了角修形 的物理意义.

2) 角修形传动蜗杆螺旋面、蜗轮齿面名义原接 触区及新接触区三者相互密切, 沿瞬时接触线奇点轨 迹互相交叉, 因此名义原接触区及新接触区各自只有 一部分保留在蜗轮齿面上. 角修形蜗杆螺旋面和蜗轮 齿面名义原接触区的密切程度, 与它和新接触区的密 切程度不同, 所以角修形能够改善名义原接触区的微

\section{表 3 算例乙的 $\boldsymbol{k}_{\rho}$ 和 $\theta_{v t}$ 的值}

\begin{tabular}{|c|c|c|c|c|c|c|c|c|}
\hline \multicolumn{2}{|c|}{ 瞬时接触线 } & \multirow{2}{*}{$\begin{array}{c}1 \\
-15.39\end{array}$} & \multirow{2}{*}{$\begin{array}{c}2 \\
-5.69\end{array}$} & \multirow{2}{*}{$\begin{array}{c}3 \\
4.01\end{array}$} & \multirow{2}{*}{$\begin{array}{c}4 \\
13.71\end{array}$} & \multirow{2}{*}{$\begin{array}{c}4^{\prime} \\
13.71 \\
\end{array}$} & \multirow{2}{*}{$\begin{array}{c}3^{\prime} \\
4.01\end{array}$} & \multirow{2}{*}{$\begin{array}{c}2^{\prime} \\
-5.69\end{array}$} \\
\hline 计算点 & $\varphi_{\mathrm{d}}\left({ }^{\circ}\right)$ & & & & & & & \\
\hline \multirow[b]{2}{*}{$a$} & $k_{\rho}$ & 0.28 & 0.35 & $\infty$ & - & - & $\infty$ & 1.33 \\
\hline & $\theta_{v t}\left({ }^{\circ}\right)$ & 43.3 & 49.0 & $(N)_{o 1}=0$ & - & - & $(N)_{o 1}=0$ & 78.9 \\
\hline \multirow{2}{*}{$b$} & $k_{\rho}$ & - & 0.29 & 0.61 & $\infty$ & $\infty$ & 3.06 & 0.83 \\
\hline & $\theta_{v t}\left({ }^{\circ}\right)$ & - & 43.2 & 49.5 & $(N)_{o 1}=0$ & $(N)_{o 1}=0$ & 53.2 & 68.5 \\
\hline \multirow[b]{2}{*}{$c$} & $k_{\rho}$ & - & 0.27 & 0.36 & - & - & 1.14 & 0.68 \\
\hline & $\theta_{v t}\left({ }^{\circ}\right)$ & - & 43.4 & 45.4 & - & - & 53.3 & 67.4 \\
\hline
\end{tabular}

\section{表 4 算例丁的 $\boldsymbol{k}_{\rho}$ 和 $\theta_{v t}$ 的值}

\begin{tabular}{|c|c|c|c|c|c|c|}
\hline \multicolumn{2}{|c|}{ 瞬时接触线 } & 1 & 2 & 3 & $3^{\prime}$ & $2^{\prime}$ \\
\hline 计算点 & $\varphi_{\mathrm{d}}\left({ }^{\circ}\right)$ & -13 & -1 & 11 & 11 & -1 \\
\hline \multirow{2}{*}{$a$} & $k_{\rho}$ & 0.21 & 0.20 & 0.19 & - & 2.69 \\
\hline & $\theta_{v t}\left({ }^{\circ}\right)$ & 42.6 & 50.2 & 58.5 & - & 66.7 \\
\hline \multirow{2}{*}{$b$} & $k_{\rho}$ & 0.19 & 0.20 & 0.2 & $\infty$ & 1.01 \\
\hline & $\theta_{v t}\left({ }^{\circ}\right)$ & 42.7 & 45.0 & 53.3 & $(N)_{o 1}=0$ & 61.4 \\
\hline \multirow{2}{*}{$c$} & $k_{\rho}$ & 0.19 & 0.19 & 0.2 & 1.79 & 0.69 \\
\hline & $\theta_{v t}\left({ }^{\circ}\right)$ & 47.3 & 44.4 & 49.1 & 36 & 60.7 \\
\hline
\end{tabular}


观啮合质量, 但新接触区的微观啮合质量, 相对于标 准传动, 变动不大, 不过仍好于名义原接触区.

3) 与标准传动不同, 角修形传动蜗轮齿面瞬时 接触线奇点轨迹不再是名义原接触线族的包络线, 名义原接触线与蜗轮齿面瞬时接触线奇点轨迹相交 于二次包络二界共轭点. 二次包络二界共轭点一侧 的名义原接触线, 在蜗轮齿面上保留下来, 形成名义 原接触区; 另一侧的名义原接触线 (对应于标准传动 蜗杆螺旋面单线工作区和蜗轮齿面二次接触区), 进 入蜗杆实体, 不能参与角修形传动副的实际啮合. 因
此, 角修形可以切去蜗轮齿面的二次接触区, 但角修 形蜗杆只能部分参与啮合.

角修正双圆环面二包传动，由于蜗杆边齿顶变尖 与根切的问题不突出, 可以应用于多头数、小传动比 的场合, 另外也有利于对传动副啮合性能进行优化; 蜗轮齿面二次接触区被切去, 整个共轭区覆盖大部分 蜗轮齿面且和蜗杆螺旋面密切; 蜗轮齿面上瞬时接触 线分布均匀、稀疏、无交叉, 蜗杆双线工作长度长; 该 传动副的诱导主曲率和滑动角数值合理, 有利于降低 接触应力水平, 形成弹流润滑油膜及增加油膜厚度.

\section{参考文献}

1 Zhao Y, Dong X, Wei W, et al. Study on the tooth surface configuration of dual tori double-enveloping worm pair without tooth flank modification. In: Proceeding of ICMT'2006, September, 2006, Chongqing, China. 180-184

2 Zhao Y, Wei W, Dong X. Tooth thickness of dual toroi enveloping toroidal worm. In: Proc. of the 12th World Congress in Mechanism and Machine Science, June, 2007, Besançon, France. 133-138

3 赵亚平. 双圆环面二次包络环面蜗杆传动的啮合界线与蜗杆螺旋面的结构. 武汉科技大学学报, 2008, 31(1): 74一 77

4 酒井高男, 牧充. 交错轴齿轮传动中第二次作用的研究, 见: 国际齿轮装置与传动会议论文选. 北京: 机械工业出版社, 1977

5 吴大任, 骆家舜. 齿轮啮合理论. 北京: 科学出版社, 1985

6 吴晓玲. 角修正可展弧面蜗杆传动的啮合分析. 齿轮, 1978(4): 48-62

7 陈惟荣. 齿轮啮合理论. 北京: 煤炭工业出版社, 1985

8 胡来瑢. 空间啮合原理及应用(下). 北京：煤炭工业出版社, 1988

9 田村久司, 酒井高男, 牧充. 以圆雉面为媒介齿轮齿面的弧面蜗杆(第一报: 齿形论的研究). 见: 酒井高男论文选集. 重庆: 重庆大 学, 1981

10 董学朱. 环面蜗杆传动设计和修形. 北京: 机械工业出版社, 2004

11 董学朱. 齿轮啮合理论基础. 北京: 机械工业出版社, 1989

12 陈志新. 共轭曲面原理(上). 北京: 科学出版社, 1974

13 齐麟, 张亚雄, 黎上威, 等. 蜗杆传动设计. 北京: 机械工业出版社, 1987 